\title{
STOCHASTIC POWER LAW FLUIDS: EXISTENCE AND UNIQUENESS OF WEAK SOLUTIONS
}

\author{
By YUtAKa Terasawa AND Nobuo YoshidA ${ }^{1}$ \\ University of Tokyo and Kyoto University
}

\begin{abstract}
We consider a stochastic partial differential equation (SPDE) which describes the velocity field of a viscous, incompressible non-Newtonian fluid subject to a random force. Here the extra stress tensor of the fluid is given by a polynomial of degree $p-1$ of the rate of strain tensor, while the colored noise is considered as a random force. We investigate the existence and the uniqueness of weak solutions to this SPDE.
\end{abstract}

1. The power law fluids. We consider a viscous, incompressible fluid whose motion is subject to a random force. The container of the fluid is supposed to be the torus $\mathbb{T}^{d}=(\mathbb{R} / \mathbb{Z})^{d} \cong[0,1]^{d}$ as a part of idealization. For a differentiable vector field $v: \mathbb{T}^{d} \rightarrow \mathbb{R}^{d}$, which is interpreted as the velocity field of the fluid, we denote the rate of strain tensor by

$$
e(v)=\left(\frac{\partial_{i} v_{j}+\partial_{j} v_{i}}{2}\right): \mathbb{T}^{d} \rightarrow \mathbb{R}^{d} \otimes \mathbb{R}^{d} .
$$

We assume that the extra stress tensor

$$
\tau(v): \mathbb{T}^{d} \rightarrow \mathbb{R}^{d} \otimes \mathbb{R}^{d}
$$

depends on $e(v)$ polynomially. More precisely, for $v>0$ (the kinematic viscosity) and $p>1$,

$$
\tau(v)=2 v\left(1+|e(v)|^{2}\right)^{(p-2) / 2} e(v) .
$$

The linearly dependent case $p=2$ is the Newtonian fluid which is described by the Navier-Stokes equations, the special case of (1.3) and (1.4). On the other hand, both the shear thinning $(p<2)$ and the shear thickening $(p>2)$ cases are considered in many fields in science and engineering. For example, shear thinning fluids are used for automobile engine oil and pipeline for crude oil transportation, while applications of shear thickening fluids can be found in modeling of body armors and automobile four wheel driving systems.

Received February 2010; revised July 2010.

${ }^{1}$ Supported in part by JSPS Grant-in-Aid for Scientific Research, Kiban (C) 21540125.

MSC2010 subject classifications. Primary 60H15; secondary 76A05, 76D05.

Key words and phrases. Stochastic partial differential equation, power law fluids. 
Given an initial velocity $u_{0}: \mathbb{T}^{d} \rightarrow \mathbb{R}^{d}$, the dynamics of the fluid are described by the following SPDE:

$$
\begin{aligned}
\operatorname{div} u & =0, \\
\partial_{t} u+(u \cdot \nabla) u & =-\nabla \Pi+\operatorname{div} \tau(u)+\partial_{t} W,
\end{aligned}
$$

where

$$
u \cdot \nabla=\sum_{j=1}^{d} u_{j} \partial_{j} \quad \text { and } \quad \operatorname{div} \tau(u)=\left(\sum_{j=1}^{d} \partial_{j} \tau_{i j}(u)\right)_{i=1}^{d} .
$$

The unknown processes in the SPDE are the velocity field $u=u(t, x)=$ $\left(u_{i}(t, x)\right)_{i=1}^{d}$ and the pressure $\Pi=\Pi(t, x)$. The Brownian motion $W=W(t, x)=$ $\left(W_{i}(t, x)\right)_{i=1}^{d}$ with values in $L_{2}\left(\mathbb{T}^{d} \rightarrow \mathbb{R}^{d}\right)$ (the set of vector fields on $\mathbb{T}^{d}$ with $L_{2}$ components) is added as the random force. Physical interpretations of (1.3) and (1.4) are the mass conservation and the motion equation, respectively. We note that the SPDE (1.3) and (1.4) for the case $p=2$ is the stochastic Navier-Stokes equation $[2,3]$.

Our motivation comes from works by Málek et al. [5], where the deterministic equation [the colored noise $\partial_{t} W$ in (1.3) and (1.4) is replaced by a nonrandom external force] is investigated. Let

$$
\begin{aligned}
& p_{1}(d)=\frac{3 d}{d+2} \vee \frac{3 d-4}{d}= \begin{cases}\frac{3 d}{d+2}, & \text { for } d \leq 4, \\
\frac{3 d-4}{d}, & \text { for } d \geq 4,\end{cases} \\
& p_{2}(d)=\frac{2 d}{d-2}, \quad p_{3}(d)=\frac{3 d-8+\sqrt{9 d^{2}+64}}{2 d}
\end{aligned}
$$

and

$$
p \in \begin{cases}\left(p_{1}(d), \infty\right), & \text { if } 2 \leq d \leq 8, \\ \left(p_{1}(9), p_{2}(9)\right) \cup\left(p_{3}(9), \infty\right), & \text { if } d=9, \\ \left(p_{3}(d), \infty\right), & \text { if } d \geq 10 .\end{cases}
$$

For example, $p_{1}(d)=\frac{3}{2}, \frac{9}{5}, 2, \frac{11}{5}$ for $d=2,3,4,5$. A basic existence theorem ([5], Theorem 3.4, page 222) states that the deterministic equation has a weak solution if (1.8) is satisfied, while a weak solution is unique if $p \geq 1+\frac{d}{2}$ ([5], Theorem 4.29, page 254).

The results in the present paper (Theorems 2.1.3 and 2.2.1) confirm that the above-mentioned deterministic results are stable under the random perturbation we consider.

Let us briefly sketch the outline of the proof of our existence result.

Step 1. Set up a finite-dimensional subspace of a smooth, divergence-free vector field, say $\mathcal{V}_{n}$, and an approximating equation to the SPDE (1.3) and (1.4) in $\mathcal{V}_{n}$. 
The good news here is that the approximating equation is a well posed stochastic differential equation (SDE) admitting a unique strong solution $u^{n} \in \mathcal{V}_{n}$. See Theorem 3.1.1 for detail.

Step 2. Establish some a priori bounds for the solution $u^{n} \in \mathcal{V}_{n}$ of the approximating SDE [e.g., (3.10), (3.13), (3.14) and (3.15)]. The point here is that the bounds should be uniform in $n$ for them to be useful. Martingale inequalities (e.g., the Burkholder-Davis-Gundy inequality) are effectively used here, working in team with the Sobolev imbedding theorem. See, for example, the proof of (3.10) for details.

Step 3. Show that the solutions $u^{n} \in \mathcal{V}_{n}$ to the approximating SDE are tight as $n \rightarrow \infty$. This is where the a priori bounds in step 2 play their roles as the moment estimates to ensure that the tails of the solutions are thin enough in certain Sobolev norms. This tightness argument is implemented in Section 3.4.

Step 4. By step $3, u^{n}(n \rightarrow \infty)$ converges in law along a subsequence to a limit. We verify that the limit is a weak solution to the SPDE (1.3) and (1.4). These will be the subjects of Section 4.1.

Here are some comments concerning the technical difference between the Navier-Stokes equations $(p=2)$ and the power law fluids. For the Navier-Stokes equations (both stochastic [2,3] and deterministic [7]), it is reasonable to discuss solutions in the $L_{2}$-space. On the other hand, for the power law fluids given by (1.2), it is the $L_{p}$-space and its dual space that become relevant. Also, due to the extra nonlinearity introduced by (1.2), some of the arguments for $p \neq 2$ become considerably more involved than the case of $p=2$, especially for $p<2$. (See, e.g., proof of Lemma 3.2.2.) We will overcome this difficulty by carrying the ideas in [5] over to the framework of Itô's calculus.

1.1. A weakformulation. Let $\mathcal{V}$ be the set of $\mathbb{R}^{d}$-valued divergence free, meanzero trigonometric polynomials, that is, the set of $v: \mathbb{T}^{d} \rightarrow \mathbb{R}^{d}$ of the following form:

$$
v(x)=\sum_{z \in \mathbb{Z}^{d} \backslash\{0\}} \widehat{v}_{z} \psi_{z}(x), \quad x \in \mathbb{T}^{d},
$$

where $\psi_{z}(x)=\exp (2 \pi \mathbf{i} z \cdot x)$ and the coefficients $\widehat{v}_{z} \in \mathbb{C}^{d}, z \in \mathbb{Z}^{d}$ satisfy

$$
\begin{aligned}
\widehat{v}_{z} & =0 \quad \text { except for finitely many } z, \\
\widehat{\widehat{v}}_{z} & =\widehat{v}_{-z} \quad \text { for all } z, \\
z \cdot \widehat{v}_{z} & =0 \quad \text { for all } z .
\end{aligned}
$$

Note that (1.12) implies that

$$
\operatorname{div} v=0 \quad \text { for all } v \in \mathcal{V} .
$$


For $\alpha \in \mathbb{R}$ and $v \in \mathcal{V}$ we define

$$
(1-\Delta)^{\alpha / 2} v=\sum_{z \in \mathbb{Z}^{d}}\left(1+4 \pi^{2}|z|^{2}\right)^{\alpha / 2} \widehat{v}_{z} \psi_{z} .
$$

We equip the torus $\mathbb{T}^{d}$ with the Lebesgue measure. For $p \in[1, \infty)$ and $\alpha \in \mathbb{R}$, we introduce

$$
V_{p, \alpha}=\text { the completion of } \mathcal{V} \text { with respect to the norm }\|\cdot\|_{p, \alpha},
$$

where

$$
\|v\|_{p, \alpha}^{p}=\int_{\mathbb{T}^{d}}\left|(1-\Delta)^{\alpha / 2} v\right|^{p}
$$

Then,

$$
V_{p, \alpha+\beta} \subset V_{p, \alpha} \quad \text { for } 1 \leq p<\infty, \alpha \in \mathbb{R} \text { and } \beta>0
$$

and the inclusion $V_{p, \alpha+\beta} \rightarrow V_{p, \alpha}$ is compact if $1<p<\infty$ ([6], (6.9), page 23).

For $v, w: \mathbb{T}^{d} \rightarrow \mathbb{R}^{d}$, with $w$ supposed to be differentiable (for a moment), we define a vector field

$$
(v \cdot \nabla) w=\sum_{j} v_{j} \partial_{j} w
$$

which is bilinear in $(v, w)$. Later on, we will generalize the definition of the above vector field; cf. (1.31).

Here are integration-by-parts formulae with which we reformulate (1.3) and (1.4) into its weak formulation. In what follows, the bracket $\langle u, v\rangle$ stands for the inner product of $L_{2}\left(\mathbb{T}^{d} \rightarrow \mathbb{R}^{d}\right)$, or its appropriate generalization, for example, the pairing of $u \in V_{p, \alpha}$ and $u \in V_{p^{\prime},-\alpha}\left(p \in(1, \infty), p^{\prime}=\frac{p}{p-1}, \alpha \geq 0\right)$. We let $C^{r}\left(\mathbb{T}^{d} \rightarrow \mathbb{R}^{d}\right)(r=1, \ldots, \infty)$ denote the set of vector fields on $\mathbb{T}^{d}$ with $C^{r}$ components.

LEMMA 1.1.1. For $v \in \mathcal{V}$ and $w, \varphi \in C^{1}\left(\mathbb{T}^{d} \rightarrow \mathbb{R}^{d}\right)$,

$$
\langle\varphi,(v \cdot \nabla) w\rangle=-\langle w,(v \cdot \nabla) \varphi\rangle .
$$

In particular,

$$
\langle w,(v \cdot \nabla) w\rangle=0
$$

Furthermore,

$$
\langle\varphi, \operatorname{div} \tau(v)\rangle=-\langle\tau(v), e(\varphi)\rangle
$$

Proof. Since $\operatorname{div} v=0$, we have that

$$
\sum_{j} \partial_{j}\left(\varphi_{i} v_{j}\right)=\sum_{j}\left(\left(\partial_{j} \varphi_{i}\right) v_{j}+\varphi_{i} \partial_{j} v_{j}\right)=\sum_{j}\left(\partial_{j} \varphi_{i}\right) v_{j} .
$$


Therefore,

$$
\begin{aligned}
\operatorname{LHS} \text { of }(1.17) & =\sum_{i, j}\left\langle\varphi_{i}, v_{j} \partial_{j} w_{i}\right\rangle=-\sum_{i, j}\left\langle\partial_{j}\left(\varphi_{i} v_{j}\right), w_{i}\right\rangle \\
& \stackrel{(1)}{=}-\sum_{i, j}\left\langle\left(\partial_{j} \varphi_{i}\right) v_{j}, w_{i}\right\rangle=\operatorname{RHS} \text { of }(1.17) .
\end{aligned}
$$

Also, by integration by parts and the symmetry of $\tau_{i j}$,

$$
\text { LHS of }(1.19)=-\sum_{i, j}\left\langle\partial_{j} \varphi_{i}, \tau_{i j}(v)\right\rangle=-\sum_{i, j}\left\langle e_{i j}(\varphi), \tau_{i j}(v)\right\rangle=\operatorname{RHS} \text { of (1.19). }
$$

Let us formally explain how the transformation of the problem (1.3) and (1.4) into its weak formulation is achieved. Suppose that $u, \Pi$ and " $\partial_{t} W$ " in (1.3) and (1.4) are regular enough. Then, for a test function $\varphi \in \mathcal{V}$,

$$
\begin{aligned}
& \partial_{t}\langle\varphi, u\rangle=-\underbrace{\langle\varphi,(u \cdot \nabla) u\rangle}_{(1)}+\underbrace{\langle\varphi, \operatorname{div} \tau(u)\rangle}_{(2)}-\underbrace{\langle\varphi, \nabla \Pi\rangle}_{(3)}+\left\langle\partial_{t} W, \varphi\right\rangle, \\
& (1) \stackrel{(1.17)}{=}-\langle(u \cdot \nabla) \varphi, u\rangle,(2) \stackrel{(1.19)}{=}-\langle e(\varphi), \tau(u)\rangle,(3)=-\langle\operatorname{div} \varphi, \Pi\rangle=0 .
\end{aligned}
$$

Thus, $(*)$ becomes

$$
\partial_{t}\langle\varphi, u\rangle=\langle(u \cdot \nabla) \varphi, u\rangle-\langle e(\varphi), \tau(u)\rangle+\partial_{t}\langle\varphi, W\rangle .
$$

By integration, we arrive at

$$
\left\langle\varphi, u_{t}\right\rangle=\left\langle\varphi, u_{0}\right\rangle+\int_{0}^{t}\left(\left\langle\left(u_{s} \cdot \nabla\right) \varphi, u_{s}\right\rangle-\left\langle e(\varphi), \tau\left(u_{s}\right)\right\rangle\right) d s+\left\langle\varphi, W_{t}\right\rangle .
$$

Here $u_{t}=u(t, \cdot)$ and $W_{t}=W(t, \cdot)$. This is a standard weak formulation of (1.3) and (1.4).

1.2. Bounds on the nonlinear terms. Let us prepare a couple of $L_{p}$-bounds on the nonlinear terms. They will be used to derive a priori bounds for the solutions later on.

LEMMA 1.2.1. Let $\alpha_{i} \in[0, \infty), p_{i} \in[1, \infty), i=1,2,3$, be such that

$$
A \geq B d, \quad \text { where } A=\sum_{i} \alpha_{i} \text { and } B=\sum_{i} \frac{1}{p_{i}}-1 \text {. }
$$

(a) Suppose (1.21) and that $\frac{\alpha_{i} B}{A}<\frac{1}{p_{i}}$ for all $i=1,2,3$. Then, there exists $C_{1} \in$ $(0, \infty)$ such that

$$
|\langle w,(v \cdot \nabla) \varphi\rangle| \leq C_{1}\|v\|_{p_{1}, \alpha_{1}}\|w\|_{p_{2}, \alpha_{2}}\|\varphi\|_{p_{3}, 1+\alpha_{3}}
$$

for $v, w, \varphi \in C^{\infty}\left(\mathbb{T}^{d} \rightarrow \mathbb{R}^{d}\right)$. 
(b) Suppose (1.21), $\alpha_{1}+\alpha_{2}>0$ and that $B \leq \frac{1}{p_{i}}$ for all $i=1,2,3$. Then, for any $\theta \in(0,1)$, there exists $C_{2} \in(0, \infty)$ such that

$$
|\langle w,(v \cdot \nabla) \varphi\rangle| \leq C_{2}\|v\|_{p_{1}, \alpha_{1}}^{\theta}\|v\|_{p_{1}, \alpha_{2}}^{1-\theta}\|w\|_{p_{2}, \alpha_{1}}^{1-\theta}\|w\|_{p_{2}, \alpha_{2}}^{\theta}\|\varphi\|_{p_{3}, 1+\alpha_{3}} .
$$

ProOF. (a) Since

$$
\sum_{i, j}\left|w_{i} v_{j} \partial_{j} \varphi_{i}\right| \leq|w||v||\nabla \varphi|
$$

we have

$$
\text { (1) }|\langle w,(v \cdot \nabla) \varphi\rangle| \leq\|v\|_{q_{1}}\|w\|_{q_{2}}\|\nabla \varphi\|_{q_{3}} \quad \text { whenever } \frac{1}{q_{1}}+\frac{1}{q_{2}}+\frac{1}{q_{3}} \leq 1 \text {. }
$$

Case 1. $B \leq 0$ : We apply (1) with $q_{i}=p_{i}(i=1,2,3)$ to get (1.22).

Case 2. $B>0$ : Since $\alpha \mapsto\|\cdot\|_{p_{i}, \alpha}$ is increasing $\left[(1-\Delta)^{-\alpha / 2}\right.$ is a contraction on $L_{p}\left(T^{d} \rightarrow \mathbb{R}^{d}\right)$ for any $\alpha \geq 0$ and $p \geq 1$ ], it is enough to prove (1.22) with $\alpha_{i}$ replaced by $\tilde{\alpha}_{i}=\frac{\alpha_{i}}{A} B d$. Therefore, we may assume without loss of generality that

$$
\max _{i} p_{i} \alpha_{i}<d \quad \text { and } \quad A=B d .
$$

We apply (1) to $q_{i} \in\left[p_{i}, \infty\right), i=1,2,3$ defined by $\frac{1}{q_{i}}=\frac{1}{p_{i}}-\frac{\alpha_{i}}{d}$. We then use the following Sobolev imbedding theorem (e.g., [6], formula (2.11), page 5). If $\alpha p<d$ and $\frac{1}{q}=\frac{1}{p}-\frac{\alpha}{d}$, then there exists $C=C(d, \alpha) \in(0, \infty)$ such that

$$
\|v\|_{q} \leq C\|v\|_{p, \alpha} \quad \text { for all } v \in C^{\infty}\left(\mathbb{T}^{d} \rightarrow \mathbb{R}^{d}\right) .
$$

(b) Let us note the following interpolation inequality (e.g., [6], formula (6.5), page 23): for any $\lambda \in[0,1]$,

(2) $\quad\|u\|_{p_{i}, \lambda \alpha_{1}+(1-\lambda) \alpha_{2}} \leq C\|u\|_{p_{i}, \alpha_{1}}^{\lambda}\|u\|_{p_{i}, \alpha_{2}}^{1-\lambda} \quad$ for $u \in V_{p_{i}, \alpha_{1}} \cap V_{p_{i}, \alpha_{2}}$.

On the other hand, we note that the assumptions for (1.22) are satisfied if we replace $\left(\alpha_{1}, \alpha_{2}\right)$ by

$$
\left(\theta \alpha_{1}+(1-\theta) \alpha_{2},(1-\theta) \alpha_{1}+\theta \alpha_{2}\right)
$$

Thus,

$$
\begin{aligned}
|\langle w,(v \cdot \nabla) \varphi\rangle| & \stackrel{(1.22)}{\leq} C_{1}\|v\|_{p_{1}, \theta \alpha_{1}+(1-\theta) \alpha_{2}}\|w\|_{p_{2},(1-\theta) \alpha_{1}+\theta \alpha_{2}}\|\varphi\|_{p_{3}, 1+\alpha_{3}} \\
& \stackrel{(2)}{\leq} \text { RHS of (1.23). }
\end{aligned}
$$

LEMMA 1.2.2. Let $\alpha \in(0,1]$ and $p \in\left(\frac{2 d}{d+2 \alpha}, \infty\right)$. 
(a) Suppose that $(d, p, \alpha) \neq(2,2,1)$. Then there exists $C_{1} \in(0, \infty)$ such that

$$
|\langle w,(v \cdot \nabla) \varphi\rangle| \leq C_{1}\|v\|_{p, \alpha}\|w\|_{2}\|\varphi\|_{p, \beta(p, \alpha)}
$$

for $v, w, \varphi \in C^{\infty}\left(\mathbb{T}^{d} \rightarrow \mathbb{R}^{d}\right)$, where

$$
\beta(p, \alpha)= \begin{cases}1+\left(\frac{2}{p}-\frac{1}{2}\right) d-\alpha>1, & \text { if } p<\frac{4 d}{d+2 \alpha}, \\ 1, & \text { if } p \geq \frac{4 d}{d+2 \alpha} .\end{cases}
$$

(b) Suppose that $d=2$. Then for any $\theta \in(0,1)$, there exists $C_{2} \in(0, \infty)$ such that

$$
|\langle w,(v \cdot \nabla) \varphi\rangle| \leq C_{2}\|v\|_{2,1}^{\theta}\|v\|_{2}^{1-\theta}\|w\|_{p, 1}^{1-\theta}\|w\|_{2}^{\theta}\|\varphi\|_{2,1}
$$

for $v, w, \varphi \in C^{\infty}\left(\mathbb{T}^{d} \rightarrow \mathbb{R}^{d}\right)$.

ProOF. We apply Lemma 1.2.1 to

$$
\left(p_{1}, p_{2}, p_{3}\right)=(p, 2, p), \quad\left(\alpha_{1}, \alpha_{2}\right)=(\alpha, 0), \quad \alpha_{3}=\left(\left(\frac{2}{p}-\frac{1}{2}\right) d-\alpha\right)^{+} .
$$

Then $\beta(p, \alpha)=1+\alpha_{3}, A=\alpha+\alpha_{3}, B=\frac{2}{p}-\frac{1}{2}$. It is enough to check that the assumptions of Lemma 1.2.1(b) are satisfied if $(d, p, \alpha)=(2,2,1)$ and that the assumptions of Lemma 1.2.1(a) are satisfied if $(d, p, \alpha) \neq(2,2,1)$. In fact, the verification for the case $(d, p, \alpha)=(2,2,1)$ can be done by simply plugging the values. We assume $(d, p, \alpha) \neq(2,2,1)$ in what follows. We may assume that $B>0$, or equivalently $p<4$. We have $A \geq B d$ by the choice of $\alpha_{i}$ 's. Let us check that

$$
\frac{\alpha_{1}}{A} B=\frac{\alpha}{\alpha+\alpha_{3}}\left(\frac{2}{p}-\frac{1}{2}\right)<\frac{1}{p} .
$$

If $(d, p, \alpha) \neq(2,2,1)$ and $p \geq \frac{4 d}{d+2 \alpha}$ (which implies $\left.p>2\right)$, then $\alpha_{3}=0$ and (1) is satisfied. If $(d, p, \alpha) \neq(2,2,1)$ and $p<\frac{4 d}{d+2 \alpha}$ (which implies $\left.p<\frac{d}{\alpha}\right)$, then $\alpha_{3}=\left(\frac{2}{p}-\frac{1}{2}\right) d-\alpha>0$. One then sees that (1) is equivalent to that $p<\frac{d}{\alpha}$ and hence, is satisfied. Let us check that

$$
\frac{\alpha_{3}}{A} B=\frac{\alpha_{3}}{\alpha+\alpha_{3}}\left(\frac{2}{p}-\frac{1}{2}\right)<\frac{1}{p} .
$$

If $(d, p, \alpha) \neq(2,2,1)$ and $p \geq \frac{4 d}{d+2 \alpha}$, then $\alpha_{3}=0$ and (2) is satisfied. If $p<\frac{4 d}{d+2 \alpha}$, then $\alpha_{3}=\left(\frac{2}{p}-\frac{1}{2}\right) d-\alpha>0$. One then sees that (2) is equivalent to that $p>\frac{2 d}{d+2 \alpha}$ and hence, is satisfied.

REMARK. We note that the following variant of (1.25) is also true:

$$
|\langle w,(v \cdot \nabla) \varphi\rangle| \leq C_{1}\|v\|_{2}\|w\|_{p, \alpha}\|\varphi\|_{p, \beta(p, \alpha)} .
$$

This can be seen by interchanging the role of $\left(p_{1}, \alpha_{1}\right)$ and $\left(p_{2}, \alpha_{2}\right)$ in the above proof. 
LEMmA 1.2.3. For $p \in(1, \infty)$, there exists $C_{1} \in(0, \infty)$ such that

$$
|\langle e(\varphi), \tau(v)\rangle| \leq C_{1}\left(1+\|e(v)\|_{p}\right)^{p-1}\|e(\varphi)\|_{p}
$$

for all $v \in V_{p, 1}$ and $\varphi \in \mathcal{V}$.

PROOF. Since

$$
|\tau(v)| \leq C(1+|e(v)|)^{p-1}
$$

we have that

$$
\begin{array}{rl}
|\langle e(\varphi), \tau(v)\rangle| & \leq C \int_{\mathbb{T}^{d}}(1+|e(v)|)^{p-1}|e(\varphi)| \\
(p-1) / p^{p+1 / p=1} & C\|1+|e(v)|\|_{p}^{p-1}\|e(\varphi)\|_{p} \\
& \leq \quad C\left(1+\|e(v)\|_{p}\right)^{p-1}\|e(\varphi)\|_{p},
\end{array}
$$

which proves (1.29).

Let $p \in\left(\frac{2 d}{d+2}, \infty\right), v, w \in V_{p, 1} \cap V_{2,0}$ and $u \in V_{p, 1}$. In view of Lemma 1.1.1, we think of $(v \cdot \nabla) w$ and $\operatorname{div} \tau(u)$, respectively, as the following linear functionals on $\mathcal{V}$ :

$$
\begin{aligned}
& \varphi \mapsto\langle\varphi,(v \cdot \nabla) w\rangle \stackrel{\text { def. }}{=}-\langle w,(v \cdot \nabla) \varphi\rangle, \\
& \varphi \mapsto\langle\varphi, \operatorname{div} \tau(u)\rangle \stackrel{\text { def. }}{=}-\langle e(\varphi), \tau(u)\rangle .
\end{aligned}
$$

Then, by Lemmas 1.2.2 and 1.2.3, they extend continuously, respectively, on $V_{p, \beta(p, 1)}$ and on $V_{p, 1}$, where

$$
\beta(p, 1)= \begin{cases}\left(\frac{2}{p}-\frac{1}{2}\right) d>1, & \text { if } p<\frac{4 d}{d+2} \\ 1, & \text { if } p \geq \frac{4 d}{d+2}\end{cases}
$$

cf. (1.26). This way, we regard $(v \cdot \nabla) w \in V_{p^{\prime},-\beta(p, 1)}\left(p^{\prime}=\frac{p}{p-1}\right)$ with

$$
\begin{aligned}
& \|(v \cdot \nabla) w\|_{p^{\prime},-\beta(p, 1)} \\
& \quad \leq \begin{cases}C\|v\|_{2,1}^{\theta}\|v\|_{2}^{1-\theta}\|w\|_{2,1}^{1-\theta}\|w\|_{2}^{\theta}, & \text { if } p=d=2, \\
C\|v\|_{p, 1}\|w\|_{2}, & \text { if otherwise, }\end{cases}
\end{aligned}
$$

and $\operatorname{div} \tau(u) \in V_{p^{\prime},-1}$ with

$$
\|\operatorname{div} \tau(u)\|_{p^{\prime},-1} \leq C\left(1+\|e(u)\|_{p}\right)^{p-1} .
$$

Finally, for $v \in V_{p, 1} \cap V_{2,0}$, we define

$$
b(v)=-(v \cdot \nabla) v+\operatorname{div} \tau(v) \in V_{p^{\prime},-\beta(p, 1)} .
$$


With this notation, (1.20) takes the form

$$
\left\langle\varphi, u_{t}\right\rangle=\left\langle\varphi, u_{0}\right\rangle+\int_{0}^{t}\left\langle\varphi, b\left(u_{s}\right)\right\rangle d s+\left\langle\varphi, W_{t}\right\rangle
$$

that is,

$$
u_{t}=u_{0}+\int_{0}^{t} b\left(u_{s}\right) d s+W_{t}
$$

as linear functionals on $\mathcal{V}$.

\section{The stochastic power law fluids.}

\subsection{The existence theorem. We need the following definition.}

DEFINITION 2.1.1. Let $H$ be a Hilbert space and $\Gamma: H \rightarrow H$ be a selfadjoint, nonnegative definite operator of trace class. A random variable $\left(W_{t}\right)_{t \geq 0}$ with values in $C([0, \infty) \rightarrow H)$ is called an $H$-valued Brownian motion with the covariance operator $\Gamma$ [abbreviated by $\operatorname{BM}(H, \Gamma)$ below] if, for each $\varphi \in H$ and $0 \leq s<t$,

$$
E\left[\exp \left(\mathbf{i}\left\langle\varphi, W_{t}-W_{s}\right\rangle\right) \mid\left(W_{u}\right)_{u \leq s}\right]=\exp \left(-\frac{t-s}{2}\langle\varphi, \Gamma \varphi\rangle\right), \quad \text { a.s. }
$$

To introduce the notion of weak solution (Definition 2.1.2), we agree on the following standard notation and convention. For a Banach space $X$, we let $L_{q, \text { loc }}([0, \infty) \rightarrow X)(1 \leq q \leq \infty)$ denote the set of locally $L_{q}$-functions $u:[0, \infty) \rightarrow X$, with the Fréchet space metric induced by the semi-norms $\|u\|_{L_{q}([0, T] \rightarrow X)}, 0<T<\infty$, where $\|u\|_{L_{q}([0, T] \rightarrow X)}$ stands for the standard $L_{q^{-}}$ norm for $\left.u\right|_{[0, T]}:[0, T] \rightarrow X$. We also regard $C([0, \infty) \rightarrow X)$, the set of continuous functions $u:[0, \infty) \rightarrow X$, as the Fréchet space induced by the semi-norms $\sup _{0 \leq t \leq T}\|u(t)\|_{X}, 0<T<\infty$.

We recall that the number $p$ is from (1.2) and that $b(v) \in V_{p^{\prime},-\beta(p, 1)}$ for $v \in$ $V_{p, 1} \cap V_{2,0}$ is defined by (1.33).

DEFINITION 2.1.2. Suppose that:

- $\Gamma: V_{2,0} \rightarrow V_{2,0}$ is a bounded self-adjoint, nonnegative definite operator of trace class;

- $\mu_{0}$ is a Borel probability measure on $V_{2,0}$;

- $(X, Y)=\left(\left(X_{t}, Y_{t}\right)\right)_{t \geq 0}$ is a process defined on a probability space $(\Omega, \mathcal{F}, P)$ such that

$$
\begin{gathered}
X \in L_{p, \operatorname{loc}}\left([0, \infty) \rightarrow V_{p, 1}\right) \cap L_{\infty, \operatorname{loc}}\left([0, \infty) \rightarrow V_{2,0}\right) \\
\cap C\left([0, \infty) \rightarrow V_{2 \wedge p^{\prime},-\beta}\right)
\end{gathered}
$$

for some $\beta>0$ and $\left(Y_{t}\right)_{t \geq 0}$ is a $\operatorname{BM}\left(V_{2,0}, \Gamma\right)$; cf. Definition 2.1.1. 
Then the process $(X, Y)$ is said to be a weak solution to the SDE

$$
X_{t}=X_{0}+\int_{0}^{t} b\left(X_{s}\right) d s+Y_{t}
$$

with the initial law $\mu_{0}$ if the following conditions are satisfied:

$$
P\left(X_{0} \in \cdot\right)=\mu_{0} ;
$$

(2.4) $Y_{t+}-Y_{t} \quad$ and $\quad\left\{\left\langle\varphi, X_{s}\right\rangle ; s \leq t, \varphi \in \mathcal{V}\right\} \quad$ are independent for any $t \geq 0$; $\left\langle\varphi, X_{t}\right\rangle=\left\langle\varphi, X_{0}\right\rangle+\int_{0}^{t}\left\langle\varphi, b\left(X_{s}\right)\right\rangle d s+\left\langle\varphi, Y_{t}\right\rangle \quad$ for all $\varphi \in \mathcal{V}$ and $t \geq 0$

We can now state our existence result.

THEOREM 2.1.3. Let $\Gamma$ and $\mu_{0}$ be as in Definition 2.1.2 and suppose additionally that:

- (1.8) holds;

- $\Delta \Gamma=\Gamma \Delta$ and both $\Gamma, \Delta \Gamma$ are of trace class;

- $\mu_{0}$ is a probability measure on $V_{2,1}$ and

$$
m_{\alpha}=\int\|\xi\|_{2, \alpha}^{2} \mu_{0}(d \xi)<\infty \quad \text { for } \alpha=0,1 .
$$

Then there exists a weak solution to the SDE (2.2) with the initial law $\mu_{0} ; c f$. Definition 2.1.2 such that (2.1) holds with $\beta=\beta(p, 1)$; $c f$. (1.30). Moreover, for any $T>0$,

$$
E\left[\sup _{t \leq T}\left\|X_{t}\right\|_{2}^{2}+\int_{0}^{T}\left\|X_{t}\right\|_{p, 1}^{p} d t\right] \leq(1+T) C<\infty,
$$

where $C=C\left(d, p, \Gamma, m_{0}\right)<\infty$.

REMARK. It would be worthwhile to mention that Theorem 2.1.3 with $p=2$ is valid for all $d$, although it is not covered by the condition (1.8) if $d \geq 4$. In fact, Lemma 3.2.2 is the only place we need condition (1.8). For $p=2$, however, we can avoid the use of that lemma; cf. remarks at the end of Section 3.4 and after Lemma 4.1.1.

2.2. The uniqueness theorem. As in the case of the deterministic equation [5], Theorem 4.29, page 254, we have the following uniqueness result:

THEOREM 2.2.1. Suppose that

$$
p \geq 1+\frac{d}{2} .
$$


Then the weak solution to the SDE (2.2), subject to the a priori bound (2.7), is pathwise unique in the following sense: if $(X, Y)$ and $(\widetilde{X}, Y)$ are two solutions on a common probability space $(\Omega, \mathcal{F}, P)$ with a common $\operatorname{BM}\left(V_{2,0}, \Gamma\right) Y$ such that $X_{0}=\tilde{X}_{0}$ a.s., then,

$$
P\left(X_{t}=\tilde{X}_{t} \text { for all } t \geq 0\right)=1 \text {. }
$$

The above uniqueness theorem, together with the Yamada-Watanabe theorem provides us with the so-called strong solution in the stochastic sense to the SDE (2.2).

Corollary 2.2.2. Suppose (2.8), in addition to all the assumptions in Theorem 2.1.3, and let $\xi$ be a given $V_{2,0}$-valued random variable with the law $\mu_{0}$ and $Y$ be a given $\operatorname{BM}\left(V_{2,0}, \Gamma\right)$ independent of $\xi$. Then there exists a process $X$ obtained as a function of $(\xi, Y)$, such that $(X, Y)$ is weak solution to the SDE (2.2) with $X_{0}=\xi$ and with all the properties stated in Theorem 2.1.3. Moreover, the law of the above process $X$ is unique.

PROOF. Corollary 2.2.2 is a direct consequence of Theorems 2.1.3 and 2.2.1 via the Yamada-Watanabe theorem [1], Theorem 1.1, page 163. The YamadaWatanabe theorem is usually stated for SDEs in finite dimensions. However, as is obvious from its proof, it applies to the present setting.

REMARK 2.2.3. For $p \in\left[1+\frac{d}{2}, \frac{2 d}{d-2}\right)$, an even stronger version of Corollary 2.2.2 is shown in [8] as a consequence of strong convergence of the Galerkin approximation; cf. Section 3.

\section{The Galerkin approximation.}

3.1. The exsitence theorem for the approximations. For each $z \in \mathbb{Z}^{d} \backslash\{0\}$, let $\left\{e_{z, j}\right\}_{j=1}^{d-1}$ be an orthonormal basis of the hyperplane $\left\{x \in \mathbb{R}^{d} ; z \cdot x=0\right\}$ and let

$$
\begin{aligned}
& \psi_{z, j}(x) \\
& \quad=\left\{\begin{array}{ll}
\sqrt{2} e_{z, j} \cos (2 \pi z \cdot x), & j=1, \ldots, d-1, \\
\sqrt{2} e_{z, j-d+1} \sin (2 \pi z \cdot x), & j=d, \ldots, 2 d-2,
\end{array} \quad x \in \mathbb{T}^{d} .\right.
\end{aligned}
$$

Then

$$
\left\{\psi_{z, j} ;(z, j) \in\left(\mathbb{Z}^{d} \backslash\{0\}\right) \times\{1, \ldots, 2 d-2\}\right\}
$$

is an orthonormal basis of $V_{2,0}$. We also introduce

$$
\begin{aligned}
& \mathcal{V}_{n}=\text { the linear span of }\left\{\psi_{z, j} ;(z, j) \text { with } z \in[-n, n]^{d}\right\} ; \\
& \mathcal{P}_{n}=\text { the orthogonal projection }: V_{2,0} \rightarrow \mathcal{V}_{n} .
\end{aligned}
$$


Using the orthonormal basis (3.1), we identify $\mathcal{V}_{n}$ with $\mathbb{R}^{N}, N=\operatorname{dim} \mathcal{V}_{n}$. Let $\mu_{0}$ and $\Gamma, V_{2,0} \rightarrow V_{2,0}$, be as in Theorem 2.1.3. Let also $\xi$ be a random variable such that $P(\xi \in \cdot)=\mu_{0}$. Finally, let $W_{t}$ be a $\operatorname{BM}\left(V_{2,0}, \Gamma\right)$ defined on a probability space $\left(\Omega^{W}, \mathcal{F}^{W}, P^{W}\right)$. Then, $\mathcal{P}_{n} W_{t}$ is identified with an $N$-dimensional Brownian motion with covariance matrix $\Gamma \mathcal{P}_{n}$. Then we consider the following approximation of (2.5):

$$
X_{t}^{n}=X_{0}^{n}+\int_{0}^{t} \mathcal{P}_{n} b\left(X_{s}^{n}\right) d s+\mathcal{P}_{n} W_{t}, \quad t \geq 0,
$$

where $X_{0}^{n}=\mathcal{P}_{n} \xi$. Let

$$
X_{t}^{n, z, j}=\left\langle X_{t}^{n}, \psi_{z, j}\right\rangle
$$

be the $(z, j)$-coordinate of $X_{t}^{n}$. Then (3.3) reads

$$
X_{t}^{n, z, j}=X_{0}^{n, z, j}+\int_{0}^{t} b^{z, j}\left(X_{s}^{n}\right) d s+W_{t}^{z, j},
$$

where

$$
\begin{aligned}
b^{z, j}\left(X_{s}^{n}\right) & =\left\langle X_{s}^{n},\left(X_{s}^{n} \cdot \nabla\right) \psi_{z, j}\right\rangle-\left\langle\tau\left(X_{s}^{n}\right), e\left(\psi_{z, j}\right)\right\rangle, \\
W_{t}^{z, j} & =\left\langle W_{t}, \psi_{z, j}\right\rangle .
\end{aligned}
$$

Let $W$. and $\xi$ be as above. We then define

$$
\begin{aligned}
\mathcal{G}_{t}^{\xi, W} & =\sigma\left(\xi, W_{s}, s \leq t\right), \quad 0 \leq t<\infty, \quad \mathcal{G}_{\infty}^{\xi, W}=\sigma\left(\bigcup_{t \geq 0} \mathcal{G}_{t}^{\xi, W}\right), \\
\mathcal{N}^{\xi, W} & =\left\{N \subset \Omega ; \exists \widetilde{N} \in \mathcal{G}_{\infty}^{\xi, W}, N \subset \widetilde{N}, P^{W}(\widetilde{N})=0\right\}
\end{aligned}
$$

and

$$
\mathcal{F}_{t}^{\xi, W}=\sigma\left(\mathcal{G}_{t}^{\xi, W} \cup \mathcal{N}^{\xi, W}\right), \quad 0 \leq t<\infty .
$$

In what follows, expectation with respect to the measure $P^{W}$ will be denoted by $E^{W}[\cdot]$.

THEOREM 3.1.1. Let $W$., $\xi$ and $\mathcal{F}_{t}^{\xi, W}$ be as above. Then for each $n=1,2, \ldots$ there exists a unique process $X^{n}$. such that:

(a) $X_{t}^{n}$ is $\mathcal{F}_{t}^{\xi, W}$-measurable for all $t \geq 0$;

(b) (3.3) is satisfied;

(c) For any $T>0$,

$$
\begin{aligned}
E^{W}\left[\left\|X_{T}^{n}\right\|_{2}^{2}+2 \int_{0}^{T}\left\langle e\left(X_{t}^{n}\right), \tau\left(X_{t}^{n}\right)\right\rangle d t\right] & =E^{W}\left[\left\|X_{0}^{n}\right\|_{2}^{2}\right]+\operatorname{tr}\left(\Gamma \mathcal{P}_{n}\right) T, \\
E^{W}\left[\left\|X_{T}^{n}\right\|_{2}^{2}+\frac{1}{C} \int_{0}^{T}\left\|X_{t}^{n}\right\|_{p, 1}^{p} d t\right] & \leq m_{0}+(C+\operatorname{tr}(\Gamma)) T<\infty,
\end{aligned}
$$

where $C=C(d, p) \in(0, \infty)$. 
Suppose, in addition, that $p \geq \frac{2 d}{d+2}$, where $p$ is from (1.2). Then, for any $T>0$,

$$
E^{W}\left[\sup _{t \leq T}\left\|X_{t}^{n}\right\|_{2}^{2}+\int_{0}^{T}\left\|X_{t}^{n}\right\|_{p, 1}^{p} d t\right] \leq(1+T) C^{\prime}<\infty,
$$

where $C^{\prime}=C^{\prime}\left(d, p, \Gamma, m_{0}\right) \in(0, \infty)$.

PROOF. We fix the accuracy $n$ of the approximation introduced above and suppress the superscript " $n$ " from the notation $X=X^{n}$. We write the summation over $z \in[-n, n]^{d}$ and $j=1, \ldots, 2 d-2$ simply by $\sum_{z, j}$. Since $v \mapsto \mathcal{P}_{n} b(v): \mathcal{V}_{n} \rightarrow \mathcal{V}_{n}$ is locally Lipschitz continuous [see (3.6)] and

$$
\langle v, b(v)\rangle \stackrel{(1.18)}{=}-\langle e(v), \tau(v)\rangle \leq C-\frac{1}{C}\|v\|_{p, 1}^{p},
$$

where we have used [5], formula (1.11), page 196, and formula $(1.20)_{2}$, page 198, to see the second inequality. This implies that there exists a unique process $X$. with the properties (a)-(b) above, as can be seen from standard existence and uniqueness results for the SDE, for example, [1], Theorem 2.4, page 177, and Theorem 3.1, pages 178-179; cf. the remark after the proof. Note that for $\alpha=$ $0,1,2, \ldots$,

$$
\left\|\nabla^{\alpha} v\right\|_{2}^{2}=\left\langle v,(-\Delta)^{\alpha} v\right\rangle=\sum_{z, j}\left(-4 \pi^{2}|z|^{2}\right)^{\alpha}\left\langle v, \psi_{z, j}\right\rangle^{2}, \quad v \in \mathcal{V}_{n} .
$$

On the other hand, we have by Itô's formula that

$$
\left|X_{t}^{z, j}\right|^{2}=\left|X_{0}^{z, j}\right|^{2}+2 \int_{0}^{t} X_{s}^{z, j} d W_{s}^{z, j}+2 \int_{0}^{t} X_{s}^{z, j} b_{s}^{z, j}\left(X_{s}\right) d s+\left\langle\psi_{z, j}, \Gamma \psi_{z, j}\right\rangle t .
$$

Therefore,

$$
\begin{aligned}
\left\|\nabla^{\alpha} X_{t}\right\|_{2}^{2}= & \left\|\nabla^{\alpha} X_{0}\right\|_{2}^{2}+2 M_{t}+2 \int_{0}^{t}\left\langle(-\Delta)^{\alpha} X_{s}, b\left(X_{s}\right)\right\rangle d s \\
& +\operatorname{tr}\left(\Gamma(-\Delta)^{\alpha} \mathcal{P}_{n}\right) t,
\end{aligned}
$$

where

$$
M_{t}=\sum_{z, j} \int_{0}^{t}(-\Delta)^{\alpha} X_{s}^{z, j} d W_{s}^{z, j} .
$$

Here we will use (3.11) only for $\alpha=0$. The case $\alpha=1$ will be used in the proof of Lemma 3.2.3 later on. By (3.11) with $\alpha=0$,

$$
\left\|X_{t}\right\|_{2}^{2}+\frac{2}{C} \int_{0}^{t}\left\|X_{s}\right\|_{p, 1}^{p} d s \leq\left\|X_{0}\right\|_{2}^{2}+2 M_{t}+(C+\operatorname{tr}(\Gamma)) t,
$$

where $M_{t}$ in (2) is defined by (3.12) with $\alpha=0$. Since it is not difficult to see that the above $M_{t}$ is a martingale (cf. [2], proof of (10), page 60), we get (3.8) by taking expectation of the equality (3.11). Similarly, we obtain (3.9) by taking expectation 
of the inequality (2). To see (3.10), it is enough to show that there exists $\delta \in(0,1]$ such that

$$
E^{W}\left[\sup _{t \leq T}\left\|X_{t}\right\|_{2}^{2}\right] \leq(1+T) C+C E^{W}\left[\left(\int_{0}^{T}\left\|X_{t}\right\|_{p, 1}^{p} d t\right)^{\delta}\right] .
$$

To see this, we start with a bound on the quadratic variation of the martingale $M$.,

$$
\langle M\rangle_{t}=\int_{0}^{t}\left\langle\Gamma X_{s}, X_{s}\right\rangle d s \leq\|\Gamma\|_{2 \rightarrow 2} \int_{0}^{t}\left\|X_{s}\right\|_{2}^{2} d s,
$$

where $\|\Gamma\|_{2 \rightarrow 2}$ denotes the operator norm of $\Gamma: V_{2,0} \rightarrow V_{2,0}$. We now recall the Burkholder-Davis-Gundy inequality ([1], Theorem 3.1, page 110),

$$
E^{W}\left[\sup _{t \leq T}\left|M_{t}\right|^{q}\right] \leq C E^{W}\left[\langle M\rangle_{T}^{q / 2}\right] \quad \text { for } q \in(0, \infty) .
$$

We then observe that

$$
E^{W}\left[\sup _{t \leq T}\left\|X_{t}\right\|_{2}^{2}\right] \stackrel{(2)}{\leq}(1+T) C+2 E^{W}\left[\sup _{t \leq T}\left|M_{t}\right|\right]
$$

$$
\stackrel{(4)-(5)}{\leq}(1+T) C+C^{\prime} E^{W}\left[\left(\int_{0}^{T}\left\|X_{s}\right\|_{2}^{2} d s\right)^{1 / 2}\right] .
$$

This proves (3) for $p \geq 2$. We assume $p<2$ in what follows. We have

$$
e_{\ell} \stackrel{\text { def. }}{=} \inf \left\{t ;\left\|X_{t}\right\|_{2} \geq \ell\right\} \nearrow \infty, \quad \text { as } \ell \nearrow \infty,
$$

since the process $X_{t}$ does not explode. On the other hand, it is clear that the following variant of (6) is true:

$$
E^{W}\left[\sup _{t \leq T \wedge e_{\ell}}\left\|X_{t}\right\|_{2}^{2}\right] \leq(1+T) C+C E^{W}\left[\left(\int_{0}^{T \wedge e_{\ell}}\left\|X_{s}\right\|_{2}^{2} d s\right)^{1 / 2}\right] .
$$

We have by Sobolev embedding that for $v \in V_{p, 1}$,

$$
\|v\|_{2} \leq C\|v\|_{p, 1}, \quad \text { since } p \geq \frac{2 d}{d+2} .
$$

Let $\varepsilon>0, r=\frac{4}{2-p} \in(4, \infty)$ and $r^{\prime}=\frac{r}{r-1}=\frac{4}{2+p} \in(1,4 / 3)$. Then,

$$
\begin{aligned}
& \left(\int_{0}^{T \wedge e_{\ell}}\left\|X_{s}\right\|_{2}^{2} d s\right)^{1 / 2} \\
& \quad \leq \sup _{s \leq T \wedge e_{\ell}}\left\|X_{s}\right\|_{2}^{(2-p) / 2}\left(\int_{0}^{T \wedge e_{\ell}}\left\|X_{s}\right\|_{2}^{p} d s\right)^{1 / 2} \\
& \quad \stackrel{(7)}{\leq} C \sup _{s \leq T \wedge e_{\ell}}\left\|X_{s}\right\|_{2}^{(2-p) / 2}\left(\int_{0}^{T \wedge e_{\ell}}\left\|X_{s}\right\|_{p, 1}^{p} d s\right)^{1 / 2} \\
& \quad \stackrel{\text { Young }}{\leq} \frac{\varepsilon^{r} C}{r} \sup _{s \leq T \wedge e_{\ell}}\left\|X_{s}\right\|_{2}^{2}+\frac{\varepsilon^{-r^{\prime}} C}{r^{\prime}}\left(\int_{0}^{T \wedge e_{\ell}}\left\|X_{s}\right\|_{p, 1}^{p} d s\right)^{2 /(2+p)} .
\end{aligned}
$$


Since $E^{W}\left[\sup _{t \leq T \wedge e_{\ell}}\left\|X_{t}\right\|_{2}^{2}\right] \leq \ell^{2}<\infty$, we have by (6) and (8) that

$$
E^{W}\left[\sup _{t \leq T \wedge e_{\ell}}\left\|X_{t}\right\|_{2}^{2}\right] \leq(1+T) C+C E^{W}\left[\left(\int_{0}^{T \wedge e \ell}\left\|X_{t}\right\|_{p, 1}^{p} d t\right)^{2 /(2+p)}\right] .
$$

Letting $\ell \nearrow \infty$, we obtain (3).

REMARK. Unfortunately, the SDE (3.3) does not satisfy the condition (2.18) imposed in the existence theorem ([1], Theorem 2.4, page 177). However, we easily see from the proof of the existence theorem that (2.18) there can be replaced by

$$
\|\sigma(x)\|^{2}+x \cdot b(x) \leq K\left(1+|x|^{2}\right) .
$$

We have applied [1], Theorem 2.4, page 177, with this modification.

3.2. Further a priori bounds. We first prove the following general estimates which apply both to the weak solution $X$ to (2.2) and to the unique solution to (3.3).

LEMMA 3.2.1. Let $T>0$ and $X=\left(X_{t}\right)_{t \geq 0}$ be a process on a probability space $(\Omega, \mathcal{F}, P)$ such that

$$
X \in L_{p}\left([0, T] \rightarrow V_{p, 1}\right) \cap L_{\infty}\left([0, T] \rightarrow V_{2,0}\right), \quad \text { a.s. }
$$

and

$$
A_{T}=E\left[\int_{0}^{T}\left\|X_{s}\right\|_{p, 1}^{p} d s\right]<\infty, \quad B_{T}=E\left[\sup _{s \in[0, T]}\left\|X_{s}\right\|_{2}^{2}\right]<\infty .
$$

(a) For $p \in\left[\frac{2 d}{d+2}, \infty\right)$,

$$
E\left[\left(\int_{0}^{T}\left\|\left(X_{s} \cdot \nabla\right) X_{s}\right\|_{p^{\prime},-\beta(p, 1)}^{p} d s\right)^{\delta}\right] \leq C A_{T}^{\delta} B_{T}^{1-\delta}<\infty,
$$

where $\delta=\frac{p}{p+2}, p^{\prime}=\frac{p}{p-1}, \beta(p, 1)$ is defined by $(1.30)$ and $C=C(d, p) \in(0, \infty)$.

$$
E\left[\int_{0}^{T}\left\|\operatorname{div} \tau\left(X_{S}\right)\right\|_{p^{\prime},-1}^{p^{\prime}} d s\right] \leq\left(T+A_{T}\right) C^{\prime}<\infty,
$$

where $C^{\prime}=C^{\prime}(p, v) \in(0, \infty)$.

ProOF. (a) We have by (1.31) that

$$
\|(v \cdot \nabla) v\|_{p^{\prime},-\beta(p, 1)} \leq C\|v\|_{p, 1}\|v\|_{2} \quad \text { for } v \in V_{p, 1} \cap V_{2,0} .
$$


We then use (1) to see that

$$
\begin{aligned}
I & \stackrel{\text { def. }}{=} \int_{0}^{T}\left\|\left(X_{s} \cdot \nabla\right) X_{s}\right\|_{p^{\prime},-\beta(p, 1)}^{p} d s \stackrel{(1)}{\leq} C \int_{0}^{T}\left\|X_{s}\right\|_{p, 1}^{p}\left\|X_{s}\right\|_{2}^{p} d s \\
& \leq C \sup _{s \in[0, T]}\left\|X_{S}\right\|_{2}^{p} \int_{0}^{T}\left\|X_{s}\right\|_{p, 1}^{p} d s .
\end{aligned}
$$

Finally, noting that $\frac{p \delta}{1-\delta}=2$, we conclude that

$$
\begin{aligned}
E\left[I^{\delta}\right] & \leq C E\left[\sup _{s \in[0, T]}\left\|X_{s}\right\|_{2}^{p \delta}\left(\int_{0}^{T}\left\|X_{s}\right\|_{p, 1}^{p} d s\right)^{\delta}\right] \\
& \leq C E\left[\sup _{s \in[0, T]}\left\|X_{s}\right\|_{2}^{2}\right]^{1-\delta} E\left[\int_{0}^{T}\left\|X_{s}\right\|_{p, 1}^{p} d s\right]^{\delta}=C B_{T}^{1-\delta} A_{T}^{\delta} .
\end{aligned}
$$

(b)

$$
\left\|\operatorname{div} \tau\left(X_{S}\right)\right\|_{p^{\prime},-1} \stackrel{(1.29)}{\leq} C\left(1+\left\|e\left(X_{S}\right)\right\|_{p}\right)^{p-1}
$$

which implies that

$$
\left\|\operatorname{div} \tau\left(X_{S}\right)\right\|_{p^{\prime},-1}^{p^{\prime}} \leq C+C\left\|e\left(X_{S}\right)\right\|_{p}^{p}
$$

and hence, that

$$
\begin{aligned}
& E\left[\int_{0}^{T}\left\|\operatorname{div} \tau\left(X_{S}\right)\right\|_{p^{\prime},-1}^{p^{\prime}} d s\right] \\
& \quad \leq C T+C E\left[\int_{0}^{T}\left\|e\left(X_{s}\right)\right\|_{p}^{p} d s\right] \leq\left(T+A_{T}\right) C .
\end{aligned}
$$

Let $X^{n}=\left(X_{t}^{n}\right)_{t \geq 0} \in \mathcal{V}$ be the unique solution of (3.3) for the Galerkin approximation.

LemmA 3.2.2. Suppose (1.8). Then, there exist $\tilde{p} \in(1, p)$ and $\tilde{\alpha} \in(1, \infty)$ such that for each $T>0$

$$
E^{W}\left[\int_{0}^{T}\left\|X_{t}^{n}\right\|_{\tilde{p}, \tilde{\alpha}}^{\tilde{p}} d t\right] \leq C_{T}<\infty,
$$

where the constant $C_{T}$ is independent of $n$.

We will have slightly better than is stated in Lemma 3.2.2 in the course of the proof. For (i) $d=2$ and $p \geq 2$ and (ii) $d \geq 3$ and $p>p_{3}(d)$, we have that

$$
E^{W}\left[\int_{0}^{T}\left\|\Delta X_{t}^{n}\right\|_{2}^{2 p /(p+2 \lambda)} d t\right] \leq C_{T}<\infty,
$$


where $\lambda \geq 0$ is defined by (3.18) below. For $p<\frac{2 d}{d-2}$, we have that

$$
E^{W}\left[\int_{0}^{T}\left\|X_{t}^{n}\right\|_{p, \tilde{\alpha}}^{\tilde{p}} d t\right] \leq C_{T}<\infty
$$

for any $\tilde{p} \in(1, p)$ with some $\tilde{\alpha}=\tilde{\alpha}(\tilde{p})>1$.

The rest of this section is devoted to the proof of Lemma 3.2.2. We suppress the superscript $n$ from the notation. We write the summation over $z \in[-n, n]^{d}$ and $j=1, \ldots, 2 d-2$ simply by $\sum_{z, j}$. We first establish the following bounds.

LeMmA 3.2.3. Suppose that $p \in\left(\frac{3 d-4}{d}, \infty\right)$ if $d \geq 3$ and let

$$
\lambda= \begin{cases}0, & \text { if } d=2, \\ \frac{2(3-p)^{+}}{d p-3 d+4}, & \text { if } d \geq 3\end{cases}
$$

cf. [5], formula (3.47), page 236,

$$
\mathcal{J}_{t}= \begin{cases}\frac{\left\|\Delta X_{t}\right\|_{2}^{2}}{\left(1+\left\|\nabla X_{t}\right\|_{2}^{2}\right)^{\lambda}}, & \text { if } p \geq 2, \\ \frac{\left\|\Delta X_{t}\right\|_{p}^{2}}{\left(1+\left\|\nabla X_{t}\right\|_{2}^{2}\right)^{\lambda}\left(1+\left\|\nabla X_{t}\right\|_{p}\right)^{2-p}}, & \text { if } 1<p<2 .\end{cases}
$$

Then, for any $T>0$,

$$
E^{W}\left[\int_{0}^{T} \mathcal{J}_{t} d t\right] \leq C_{T}<\infty,
$$

where $C_{T}=C\left(T, d, p, \Gamma, m_{1}\right)$.

Proof. By (3.11) with $\alpha=1$,

$$
\frac{1}{2}\left\|\nabla X_{t}\right\|_{2}^{2}=\frac{1}{2}\left\|\nabla X_{0}\right\|_{2}^{2}+M_{t}+\int_{0}^{t} K_{s} d s,
$$

where

$$
M_{t}=-\sum_{z, j} \int_{0}^{t} \Delta X_{s}^{z, j} d W_{s}^{z, j}, \quad K_{s}=\left\langle-\Delta X_{s}, b\left(X_{s}\right)\right\rangle+\frac{1}{2} \operatorname{tr}\left(-\Gamma \Delta \mathcal{P}_{n}\right) .
$$

Step 1 . We will prove that

$$
K_{s}+c_{1} \mathcal{I}_{s} \leq \begin{cases}0, & \text { if } d=2, \\ C_{1}\left(1+\left\|\nabla X_{t}\right\|_{2}^{2}\right)^{\lambda}\left(1+\left\|\nabla X_{t}\right\|_{p}\right)^{p}, & \text { if } d \geq 3,\end{cases}
$$

where $c_{1}, C_{1} \in(0, \infty)$ are constants and

$$
\mathcal{I}_{s}=\int_{\mathbb{T}^{d}}\left(1+\left|e\left(X_{s}\right)\right|^{2}\right)^{(p-2) / 2}\left|\nabla e\left(X_{S}\right)\right|^{2}
$$


To show (2), note that

$$
\left\langle-\Delta X_{s}, b\left(X_{s}\right)\right\rangle=\left\langle-\Delta X_{S},\left(X_{s} \cdot \nabla\right) X_{s}\right\rangle-\left\langle\tau\left(X_{S}\right), e\left(-\Delta X_{S}\right)\right\rangle .
$$

We see from the argument in [5], proof of (3.19), page 225, that

$$
\left\langle\tau\left(X_{S}\right), e\left(-\Delta X_{S}\right)\right\rangle \geq 2 c_{1} \mathcal{I}_{s} .
$$

On the other hand, we have by integration by parts and Hölder's inequality that

$$
\left\langle-\Delta X_{s},\left(X_{s} \cdot \nabla\right) X_{s}\right\rangle=\sum_{i, j, k} \int_{\mathbb{T}^{d}} \partial_{k} X_{s}^{j} \partial_{j} X_{s}^{i} \partial_{k} X_{s}^{i} \leq\left\|\nabla X_{s}\right\|_{3}^{3},
$$

where $X_{s}^{j}=\sum_{z \in[-n, n]^{d}} X_{s}^{z, j} \psi_{z, j}$. It is also well known that the inner product on the LHS vanishes if $d=2$ ([5], formula (3.20), page 225). By the argument in [5], proof of (3.46), pages 234-235 (this is where the choice of $\lambda$ is used), we get

$$
\left\|\nabla X_{s}\right\|_{3}^{3} \leq C_{1}\left(1+\left\|\nabla X_{t}\right\|_{2}^{2}\right)^{\lambda}\left(1+\left\|\nabla X_{t}\right\|_{p}\right)^{p}+c_{1} \mathcal{I}_{s} .
$$

These imply that

$$
\begin{aligned}
& \left\langle-\Delta X_{s},\left(X_{s} \cdot \nabla\right) X_{s}\right\rangle \\
& \quad \times \begin{cases}=0, & \text { if } d=2, \\
\leq C_{1}\left(1+\left\|\nabla X_{t}\right\|_{2}^{2}\right)^{\lambda}\left(1+\left\|\nabla X_{t}\right\|_{p}\right)^{p}+c_{1} \mathcal{I}_{s}, & \text { if } d \geq 3 .\end{cases}
\end{aligned}
$$

We get (2) by (3)-(4).

Step 2. Proof of (3.20). By [5], formulas (3.25) and (3.26), page 227, $\mathcal{J}_{t}$ and $\mathcal{I}_{t}$ are related as

$$
\mathcal{J}_{t} \leq C \frac{\mathcal{I}_{t}}{\left(1+\left\|\nabla X_{t}\right\|_{2}^{2}\right)^{\lambda}} .
$$

Therefore, it is enough to prove that

$$
E^{W}\left[\int_{0}^{t} \frac{\mathcal{I}_{s} d s}{\left(1+\left\|\nabla X_{s}\right\|_{2}^{2}\right)^{\lambda}}\right] \leq C_{T}<\infty,
$$

where $C_{T}=C\left(T, d, p, \Gamma, m_{0}, m_{1}\right) \in(0, \infty)$.

To see this, we introduce the following concave function of $x \geq 0$ :

$$
f(x)= \begin{cases}\frac{1}{1-\lambda}(1+x)^{1-\lambda}, & \text { if } \lambda \neq 1, \\ \ln (1+x), & \text { if } \lambda=1 .\end{cases}
$$

Then we have by (1) and Itô's formula that

$$
f\left(\left\|\nabla X_{t}\right\|_{2}^{2}\right) \leq f\left(\left\|\nabla X_{0}\right\|_{2}^{2}\right)+\int_{0}^{t} \frac{d M_{s}}{\left(1+\left\|\nabla X_{s}\right\|_{2}^{2}\right)^{\lambda}}+2 \int_{0}^{t} \frac{K_{s} d s}{\left(1+\left\|\nabla X_{s}\right\|_{2}^{2}\right)^{\lambda}},
$$


where we have omitted the term with $f^{\prime \prime} \leq 0$. Moreover, by (2)

$$
\begin{aligned}
\frac{K_{s}}{\left(1+\left\|\nabla X_{s}\right\|_{2}^{2}\right)^{\lambda}} & \leq-\frac{c_{1} \mathcal{I}_{s}}{\left(1+\left\|\nabla X_{s}\right\|_{2}^{2}\right)^{\lambda}}+C_{1}\left(1+\left\|\nabla X_{s}\right\|_{p}\right)^{p}, \\
0 & \leq f(x) \leq C_{2}(1+x) \quad \text { if } \lambda \in[0,1]
\end{aligned}
$$

and

$$
-\frac{1}{\lambda-1} \leq f(x) \leq 0 \quad \text { if } \lambda>1
$$

Putting these together, we get

$$
\begin{aligned}
-C_{3} & +2 c_{1} E^{W}\left[\int_{0}^{t} \frac{\mathcal{I}_{s} d s}{\left(1+\left\|\nabla X_{s}\right\|_{2}^{2}\right)^{\lambda}}\right] \\
& \leq C_{2}\left(1+E\left[\left\|\nabla X_{0}\right\|_{2}^{2}\right]\right)+C_{1} E^{W}\left[\int_{0}^{t}\left(1+\left\|\nabla X_{s}\right\|_{p}\right)^{p} d s\right] \\
& \stackrel{(3.10)}{\leq} C\left(T, d, p, \Gamma, m_{0}, m_{1}\right)<\infty,
\end{aligned}
$$

where $C_{3}=0$ if $\lambda \in(0,1]$ and $C_{3}=\frac{1}{\lambda-1}$ if $\lambda>1$. This proves (5).

Proof OF LEMMA 3.2.2. We note that

$$
\begin{aligned}
& p_{1}(d)<p_{3}(d)<p_{2}(d) \quad \text { for } d \leq 8, \\
& p_{1}(9)=2.555 \ldots<p_{2}(9)=2.5714 \ldots<p_{3}(9)=2.620 \ldots, \\
& p_{2}(d)<p_{1}(d) \quad \text { for } d \geq 10 .
\end{aligned}
$$

Thus, condition (1.8) takes the following form in any $d \geq 2$ :

$$
p \in\left(p_{1}(d), p_{2}(d)\right) \cup\left(p_{3}(d), \infty\right) .
$$

We consider the following four cases separately:

Case 1. $d=2$ and $p \geq 2$;

Case 2. $d \geq 3$ and $p>p_{3}(d)$;

Case 3. $p \in\left(p_{1}(d), p_{2}(d)\right)$ and $p \geq 2$;

Case 4. $p \in\left(p_{1}(d), 2\right)$ (this case appears only if $\left.d=2,3\right)$.

The first two cases cover the interval $\left(p_{3}(d), \infty\right)$ in $(3.21)$. [Note that $p_{3}(2)=2$, while the last two cases cover the interval $\left(p_{1}(d), p_{2}(d)\right.$.]

Case 1. By (3.20), (3.15) has already been shown with $\tilde{p}=\tilde{\alpha}=2$. 
Case 2. Note that $p>p_{3}(d)>2$ and that $\beta \stackrel{\text { def. }}{=} \frac{p}{p+2 \lambda}>1 / 2$. We prove (3.16). Since $\lambda \beta=\frac{p}{2}(1-\beta)$,

$$
\begin{aligned}
& E^{W}\left[\int_{0}^{T}\left\|\Delta X_{s}\right\|_{2}^{2 \beta} d s\right] \\
& =E^{W}\left[\int_{0}^{T} \mathcal{J}_{s}^{\beta}\left(1+\left\|\nabla X_{s}\right\|_{2}^{2}\right)^{\lambda \beta} d s\right] \\
& \stackrel{\substack{(1-\beta)=1 \\
\leq}}{=} E^{W}\left[\int_{0}^{T} \mathcal{J}_{s} d s\right]^{\beta} E^{W}\left[\int_{0}^{T}\left(1+\left\|\nabla X_{s}\right\|_{2}^{2}\right)^{p / 2} d s\right]^{1-\beta} \\
& \stackrel{\text { (3.10), (3.20) }}{\leq} C_{T}<\infty \text {, }
\end{aligned}
$$

where we used (3.20) for $p \geq 2$.

Case 3. We prove (3.17) for given $\tilde{p} \in(1, p)$ with some $\tilde{\alpha}=\tilde{\alpha}(\tilde{p}) \in(1,2)$. Let $\beta=\frac{p}{p+2 \lambda} \in(0,1)$. Then the bound (1) from case 2 is still valid, although it may no longer be the case that $2 \beta>1$ here. On the other hand, it is not difficult to see via the interpolation and the Sobolev imbedding that for any $\tilde{p} \in(1, p)$, there exist $\tilde{\alpha} \in(1,2)$ and $\theta \in(0,1)$ such that

$$
\int_{0}^{T}\left\|X_{s}\right\|_{p, \tilde{\alpha}}^{\tilde{p}} d s \leq C\left(\int_{0}^{T}\left\|X_{s}\right\|_{p, 1}^{p} d s\right)^{\theta}\left(\int_{0}^{T}\left\|X_{s}\right\|_{2,2}^{2 \beta} d s\right)^{1-\theta}
$$

cf. [5], proof of (3.58), page 238. This is where the restriction $p<\frac{2 d}{d-2}$ is necessary. Thus,

$$
\begin{gathered}
E^{W}\left[\int_{0}^{T}\left\|X_{s}\right\|_{p, \tilde{\alpha}}^{\tilde{p}} d s\right] \leq C E^{W}\left[\int_{0}^{T}\left\|X_{s}\right\|_{p, 1}^{p} d s\right]^{\theta} E^{W}\left[\int_{0}^{T}\left\|X_{s}\right\|_{2,2}^{2 \beta} d s\right]^{1-\theta} \\
\stackrel{(3.10),(1)}{\leq} C_{T}<\infty .
\end{gathered}
$$

Case 4. We prove (3.17) for given $\tilde{p} \in(1, p)$ and with some $\tilde{\alpha}=\tilde{\alpha}(\tilde{p}) \in(1,2)$. We recall that $p>\frac{3 d}{d+2}$ and set

$$
\beta=\frac{((d+2) p-3 d) p}{2\left((d+5) p-3 d-p^{2}\right)} \in\left(0, \frac{1}{2}\right) .
$$

Then,

$$
\rho \stackrel{\text { def. }}{=} \frac{(2-p) d \lambda}{2(1-\beta) p} \in[0,1) \quad \text { and } \quad \frac{(2-p) \beta}{1-\beta} \in(0, p) .
$$

As a result of applications of Hölder's inequality, the interpolation and the Sobolev imbedding (cf. [5], formulas (3.60)-(3.63), pages 239-240), we arrive at the following bound:

$$
\int_{0}^{T}\left\|\Delta X_{s}\right\|_{p}^{2 \beta} d s \leq C\left(\int_{0}^{T} \mathcal{J}_{s} d s\right)^{\beta}\left(I_{1}+I_{2}\right)^{1-\beta}
$$


where

$$
\begin{aligned}
& I_{1}=\int_{0}^{T}\left(1+\left\|\nabla X_{s}\right\|_{p}\right)^{(2-p) \beta /(1-\beta)} d s, \\
& I_{2}=\left(\int_{0}^{T}\left\|\Delta X_{s}\right\|_{p}^{2 \beta} d s\right)^{\rho}\left(\int_{0}^{T}\left\|\nabla X_{s}\right\|_{p}^{p} d s\right)^{1-\rho} .
\end{aligned}
$$

We first prove that

$$
E^{W}\left[\int_{0}^{T}\left\|\Delta X_{s}\right\|_{p}^{2 \beta} d s\right] \leq C_{T}<\infty .
$$

We first assume $d=3$, where $\rho>0$. Let $r=\frac{1}{\rho} \in(1, \infty)$ and $r^{\prime}=\frac{r}{r-1}=\frac{1}{1-\rho} \in$ $(1, \infty)$. Then, for $\varepsilon>0$,

$$
\begin{aligned}
& E^{W}\left[\int_{0}^{T}\left\|\Delta X_{s}\right\|_{p}^{2 \beta} d s\right] \quad \stackrel{(3)}{\leq} \quad C E^{W}\left[\left(\int_{0}^{T} \mathcal{J}_{s} d s\right)^{\beta}\left(I_{1}+I_{2}\right)^{1-\beta}\right] \\
& \underset{\leq}{\beta+(1-\beta)=1} C E^{W}\left[\int_{0}^{T} \mathcal{J}_{s} d s\right]^{\beta} E^{W}\left[I_{1}+I_{2}\right]^{1-\beta} \\
& \stackrel{(3.20)}{\leq} \quad C_{T} E\left[1+I_{1}+I_{2}\right] \text {, } \\
& E^{W}\left[I_{1}\right] \stackrel{(3.10),(2)}{\leq} \quad C_{T}<\infty, \\
& E^{W}\left[I_{2}\right] \stackrel{\text { Young }}{\leq} \frac{\varepsilon^{r}}{r} E^{W}\left[\int_{0}^{T}\left\|\Delta X_{S}\right\|_{p}^{2 \beta} d s\right] \\
& +\frac{\varepsilon^{-r^{\prime}}}{r^{\prime}} E^{W}\left[\int_{0}^{T}\left\|\nabla X_{s}\right\|_{p}^{p} d s\right] \\
& \stackrel{\text { (3.10) }}{\leq} \frac{\varepsilon^{r}}{r} E^{W}\left[\int_{0}^{T}\left\|\Delta X_{s}\right\|_{p}^{2 \beta} d s\right]+C_{T} .
\end{aligned}
$$

Putting things together, with $\varepsilon$ small enough, we arrive at (4) for $d=3$. If $d=2$ and hence, $\rho=0$, then we have $E^{W}\left[I_{2}\right] \leq C_{T}$ directly from (3.10). Therefore, the proof of (4) is even easier than the above.

We finally turn to (3.15). It is not difficult to see via the interpolation (cf. [5], proof of (3.65), pages 240-241) that for any $\tilde{p} \in(1, p)$, there exist $\tilde{\alpha} \in(1,2)$ and $\theta \in(0,1)$ such that

$$
\int_{0}^{T}\left\|X_{s}\right\|_{p, \tilde{\alpha}}^{\tilde{p}} d s \leq C\left(\int_{0}^{T}\left\|X_{s}\right\|_{p, 1}^{p} d s\right)^{\theta}\left(\int_{0}^{T}\left\|X_{s}\right\|_{p, 2}^{2 \beta} d s\right)^{1-\theta} .
$$

Thus,

$$
\begin{gathered}
E^{W}\left[\int_{0}^{T}\left\|X_{s}\right\|_{p, \tilde{\alpha}}^{\tilde{p}} d s\right] \leq C E^{W}\left[\int_{0}^{T}\left\|X_{s}\right\|_{p, 1}^{p} d s\right]^{\theta} E^{W}\left[\int_{0}^{T}\left\|X_{s}\right\|_{p, 2}^{2 \beta} d s\right]^{1-\theta} \\
\stackrel{(3.10),(4)}{\leq} C_{T}<\infty
\end{gathered}
$$


3.3. Compact imbedding lemmas. We will need some compact imbedding lemmas from [3]. We first introduce the following definition.

Definition 3.3.1. Let $p \in[1, \infty), T \in(0, \infty)$ and $E$ be a Banach space.

(a) We let $L_{p, 1}([0, T] \rightarrow E)$ denote the Sobolev space of all $u \in L_{p}([0, T] \rightarrow E)$ such that

$$
u(t)=u(0)+\int_{0}^{t} u^{\prime}(s) d s \quad \text { for almost all } t \in[0, T]
$$

with some $u(0) \in E$ and $u^{\prime}(\cdot) \in L_{p}([0, T] \rightarrow E)$. We endow the space $L_{p, 1}([0, T] \rightarrow E)$ with the norm $\|u\|_{L_{p, 1}([0, T] \rightarrow E)}$ defined by

$$
\|u\|_{L_{p, 1}([0, T] \rightarrow E)}^{p}=\int_{0}^{T}\left(|u(t)|_{E}^{p}+\left|u^{\prime}(t)\right|_{E}^{p}\right) d t .
$$

(b) For $\alpha \in(0,1)$, we let $L_{p, \alpha}([0, T] \rightarrow E)$ denote the Sobolev space of all $u \in$ $L_{p}([0, T] \rightarrow E)$ such that

$$
\int_{0<s<t<T} \frac{|u(t)-u(s)|_{E}^{p}}{|t-s|^{1+\alpha p}} d s d t<\infty .
$$

We endow the space $L_{p, \alpha}([0, T] \rightarrow E)$ with the norm $\|u\|_{L_{p, \alpha}([0, T] \rightarrow E)}$ defined by

$$
\|u\|_{L_{p, \alpha}([0, T] \rightarrow E)}^{p}=\int_{0}^{T}|u(t)|^{p} d t+\int_{0<s<t<T} \frac{|u(t)-u(s)|_{E}^{p}}{|t-s|^{1+\alpha p}} d s d t .
$$

To introduce the compact imbedding lemmas, we agree on the following standard convention. Let $X$ be a vector space and $X_{i} \subset X$ be a subspace with the norm $\|\cdot\|_{i}(i=1,2)$. Then we equip $X_{0} \cap X_{1}$ and $X_{0}+X_{1}$, respectively, with the norms

$$
\begin{aligned}
& \|u\|_{X_{0} \cap X_{1}}=\|u\|_{0}+\|u\|_{1}, \\
& \|u\|_{X_{0}+X_{1}}=\inf \left\{\left\|u_{0}\right\|_{0}+\left\|u_{1}\right\|_{1} ; u=u_{0}+u_{1}, u_{i} \in X_{i}\right\} .
\end{aligned}
$$

The following lemmas will be used in Section 3.4.

Lemma 3.3.2 ([3], Theorem 2.2, page 370). Let:

- $E_{1}, \ldots, E_{n}$ and $E$ be Banach spaces such that each $E_{i} \stackrel{\text { compact }}{\hookrightarrow} E, i=1, \ldots, n$.

- $p_{1}, \ldots, p_{n} \in(1, \infty), \alpha_{1}, \ldots, \alpha_{n}>0$ are such that $p_{i} \alpha_{i}>1, i=1, \ldots, n$.

Then, for any $T>0$,

$$
L_{p_{1}, \alpha_{1}}\left([0, T] \rightarrow E_{1}\right)+\cdots+L_{p_{n}, \alpha_{n}}\left([0, T] \rightarrow E_{n}\right) \stackrel{\text { compact }}{\hookrightarrow} C([0, T] \rightarrow E) .
$$


Lemma 3.3.3 ([3], Theorem 2.1, page 372). Let

$$
E_{0} \stackrel{\text { compact }}{\hookrightarrow} G E_{1}
$$

be Banach spaces such that the first embedding is compact and $E_{0}, E_{1}$ are reflexive. Then, for any $p \in(1, \infty), \alpha \in(0,1)$ and $T>0$,

$$
L_{p}\left([0, T] \rightarrow E_{0}\right) \cap L_{p, \alpha}\left([0, T] \rightarrow E_{1}\right) \stackrel{\text { compact }}{\hookrightarrow} L_{p}([0, T] \rightarrow E) .
$$

3.4. Convergence of the approximations. Let $X^{n}=\left(X_{t}^{n}\right)_{t \geq 0} \in \mathcal{V}$ be the unique solution to (3.3) for the Galerkin approximation. We write

$$
p^{\prime}=\frac{p}{p-1}, \quad p^{\prime \prime}=p \wedge p^{\prime} .
$$

Let $\beta(p, 1)$ be defined by (1.30) and let $\tilde{p}>1$ be the one from Lemma 3.2.2. We may assume that $\tilde{p} \in\left(1, p^{\prime \prime}\right]$. We also agree on the following standard convention. Let $S$ be a set and $\rho_{i}$ be a metric on $S_{i} \subset S(i=1,2)$. Then we tacitly consider the metric $\rho_{1}+\rho_{2}$ on the set $S_{1} \cap S_{2}$; cf. (3.23).

PROPOSITION 3.4.1. Let $\beta>\beta(p, 1)$. Then there exist a process $X$ and a sequence $\left(\widetilde{X}^{k}\right)_{k \geq 1}$ of processes defined on a probability space $(\Omega, \mathcal{F}, P)$ such that the following properties are satisfied:

(a) The process $X$ takes values in

$$
C\left([0, \infty) \rightarrow V_{2 \wedge p^{\prime},-\beta}\right) \cap L_{\tilde{p}, \mathrm{loc}}\left([0, \infty) \rightarrow V_{\tilde{p}, 1}\right) .
$$

(b) For some sequence $n(k) \nearrow \infty, \widetilde{X}^{k}$ has the same law as $X^{n(k)}$ and

$$
\lim _{k \rightarrow \infty} \widetilde{X}^{k}=X \text { in the metric space (3.23), P-a.s. }
$$

REMARKS. (1) Due to Skorohod's representation theorem used in Lemma 3.4.5 below, the probability space $(\Omega, \mathcal{F}, P)$ in the above proposition may not be the same as $\left(\Omega^{W}, \mathcal{F}^{W}, P^{W}\right)$, where we have solved the SDE (3.3).

(2) See (4.4) below for additional information on the convergence (3.24).

We divide the Proposition 3.4.1 into Lemmas 3.4.3-3.4.5. To prepare the proofs of these lemmas, we write (3.3) as

$$
X_{t}^{n}=X_{0}^{n}+I_{t}^{n}+J_{t}^{n}+W_{t}^{n},
$$

with

$$
\begin{aligned}
I_{t}^{n} & =\int_{0}^{t} \mathcal{P}_{n}\left(\left(X_{s}^{n} \cdot \nabla\right) X_{s}^{n}\right) d s, \quad J_{t}^{n}=\int_{0}^{t} \mathcal{P}_{n}\left(\operatorname{div} \tau\left(X_{s}^{n}\right)\right) d s, \\
W_{t}^{n} & =\mathcal{P}_{n} W_{t} .
\end{aligned}
$$


It is elementary to obtain the following regularity bound of the noise term $W_{t}^{n}$ [2], Corollary 4.2, page 92: for any $p \in[1, \infty), \alpha \in[0,1 / 2)$ and $T>0$, there exists $C_{T}=C_{\alpha, p, T} \in(0, \infty)$ such that

$$
\sup _{n \geq 0} E^{W}\left[\left\|W^{n}\right\|_{L_{p, \alpha}\left([0, T] \rightarrow V_{2,0}\right)}^{p}\right] \leq C_{T} \operatorname{tr}(\Gamma)^{p / 2} .
$$

We will control $I^{n}$ and $J_{.}^{n}$ by (3.13) and (3.14). However, to be able to do so, we have to get rid of the projection $\mathcal{P}_{n}$. This is the content of the following:

LemMA 3.4.2. Let $T \in(0, \infty)$. Then,

$$
\sup _{n \geq 1} E^{W}\left[\left\|I^{n}\right\|_{L_{p, 1}\left([0, T] \rightarrow V_{p^{\prime},-\beta(p, 1)}\right)}^{\gamma}\right] \leq C_{T}<\infty,
$$

where $\gamma=\frac{p^{2}}{p+2}$. Also,

$$
\sup _{n \geq 1} E^{W}\left[\left\|J^{n}\right\|_{L_{p^{\prime}, 1}\left([0, T] \rightarrow V_{p^{\prime},-\beta(p, 1)}\right)}^{p^{\prime}}\right] \leq C_{T}<\infty .
$$

ProOf. For any $p \in(1, \infty)$, there exists $A_{p} \in(0, \infty)$ such that

$$
\left\|\mathcal{P}_{n} v\right\|_{p} \leq A_{p}\|v\|_{p} \quad \text { for all } v \in V_{p, 0}
$$

(See, e.g., [4], Theorem 3.5.7, page 213.) This implies that $\left\|\mathcal{P}_{n} v\right\|_{p, \alpha} \leq A_{p}\|v\|_{p, \alpha}$ and hence, $\left\|\mathcal{P}_{n} v\right\|_{p^{\prime},-\alpha} \leq A_{p}\|v\|_{p^{\prime},-\alpha}$ for any $p \in(1, \infty)$ and $\alpha \geq 0$. We combine this and (3.13) and (3.14) to obtain (3.27) and (3.28).

LEMMA 3.4.3. For $\beta>\beta(p, 1)$, the laws $\left\{P^{W}\left(X^{n} \in \cdot\right)\right\}_{n=1}^{\infty}$ are tight on $C\left([0, \infty) \rightarrow V_{2 \wedge p^{\prime},-\beta}\right)$.

PROOF. As is easily seen, it is enough to prove the following:

(1) The laws $\left\{P^{W}\left(\left(X_{t}^{n}\right)_{t \leq T} \in \cdot\right)\right\}_{n=1}^{\infty}$ are tight on $C\left([0, T] \rightarrow V_{2 \wedge p^{\prime},-\beta}\right)$ for each fixed $T>0$. To see (1), we set

$$
\begin{aligned}
\mathcal{S}= & L_{p, 1}\left([0, T] \rightarrow V_{p^{\prime},-\beta(p, 1)}\right)+L_{p^{\prime}, 1}\left([0, T] \rightarrow V_{p^{\prime},-1}\right) \\
& +L_{2 / \gamma, \gamma}\left([0, T] \rightarrow V_{2,0}\right), \quad \text { with } \gamma \in(0,1 / 2) .
\end{aligned}
$$

We then see from Lemma 3.3.2 that

$$
\mathcal{S} \stackrel{\text { compact }}{\hookrightarrow} C\left([0, T] \rightarrow V_{2 \wedge p^{\prime},-\beta}\right) .
$$

On the other hand, we have that

$$
\sup _{n} E^{W}\left[\left\|I_{.}^{n}\right\|_{L_{p, 1}\left([0, T] \rightarrow V_{p^{\prime},-\beta(p, 1)}\right)}^{\delta} \stackrel{(3.27)}{\leq} C_{T}<\infty \quad \text { for some } \delta \in(0,1]\right.
$$

$\sup _{n} E^{W}\left[\left\|J^{n}\right\|_{L_{p^{\prime}, 1}\left([0, T] \rightarrow V_{p^{\prime},-1}\right)} \stackrel{(3.28)}{\leq} C_{T}<\infty\right.$;

(5) $\sup _{n} E^{W}\left[\left\|X_{0}^{n}+W^{n}\right\|_{L_{2 / \gamma, \gamma}\left([0, T] \rightarrow V_{2,0}\right)}\right] \stackrel{(3.26)}{\leq} C_{T}<\infty$. 
We conclude from (3)-(5) and (3.25) that

$$
\sup _{n} E^{W}\left[\left\|X^{n}\right\|_{\mathcal{S}}^{\delta}\right] \leq C_{T}<\infty
$$

and hence, that for $R>0$,

$$
\begin{aligned}
\sup _{n} P^{W}\left(\left\|X^{n}\right\|_{\mathcal{S}}>R\right) & \leq \frac{1}{R^{\delta}} \sup _{n} E^{W}\left[\left\|X^{n}\right\|_{\mathcal{S}}^{\delta}\right] \\
& \leq \frac{C_{T}}{R^{\delta}} \longrightarrow 0 \quad \text { as } R \longrightarrow \infty
\end{aligned}
$$

We see from (2) that the set

$$
\left\{X ;\left\|X^{n}\right\|_{\mathcal{S}} \leq R\right\}
$$

is relatively compact in $C\left([0, T] \rightarrow V_{2 \wedge p^{\prime},-\beta}\right)$. Hence, by (6), we have the tightness (1).

LEMMA 3.4.4. The laws $\left\{P^{W}\left(X^{n} \in \cdot\right)\right\}_{n=1}^{\infty}$ are tight on $L_{\tilde{p}, \text { loc }}([0, \infty) \rightarrow$ $\left.V_{\tilde{p}, 1}\right)$.

Proof. Let $\tilde{p}>1$ and $\tilde{\alpha}>1$ be from Lemma 3.2.2. We may assume that $\tilde{p} \in\left(1, p^{\prime \prime}\right]$. It is enough to prove the following:

The laws $\left\{P^{W}\left(\left(X_{t}^{n}\right)_{t \leq T} \in \cdot\right)\right\}_{n=1}^{\infty}$ are tight on $L_{\tilde{p}}\left([0, T] \rightarrow V_{\tilde{p}, 1}\right)$

for each fixed $T>0$.

To see (1), we set

$$
\mathcal{I}=L_{\tilde{p}}\left([0, T] \rightarrow V_{\tilde{p}, \tilde{\alpha}}\right) \cap L_{\tilde{p}, \gamma}\left([0, T] \rightarrow V_{\tilde{p},-\beta(p, 1)}\right) \quad \text { with } \gamma \in(0,1 / 2) .
$$

Note that

$$
V_{\tilde{p}, \tilde{\alpha}} \stackrel{\text { compact }}{\hookrightarrow} V_{\tilde{p}, 1} \hookrightarrow V_{\tilde{p},-\beta(p, 1)}
$$

and hence, by Lemma 3.3.3, that

$$
\mathcal{I} \stackrel{\text { compact }}{\hookrightarrow} L_{\tilde{p}}\left([0, T] \rightarrow V_{\tilde{p}, 1}\right) .
$$

On the other hand,

$$
\sup _{n} E^{W}\left[\left\|X^{n}\right\|_{L_{\tilde{p}}\left([0, T] \rightarrow V_{\tilde{p}, \tilde{\alpha}}\right)} \stackrel{(3.15)}{\leq} C_{T}<\infty .\right.
$$

Moreover, for some $\delta \in(0,1]$,

$$
\begin{aligned}
& \sup _{n} E^{W}\left[\left\|X_{.}^{n}\right\|_{L_{\tilde{p}, \gamma}}^{\delta}\left([0, T] \rightarrow V_{\tilde{p},-\beta(p, 1)}\right)\right] \\
& \leq \sup _{n} E^{W}\left[\left\|X_{0}^{n}+I^{n}+J^{n}\right\|_{L_{\tilde{p}, \gamma}}^{\delta}\left([0, T] \rightarrow V_{\tilde{p},-\beta(p, 1)}\right)\right] \\
& +\sup _{n} E^{W}\left[\left\|W^{n}\right\|_{L_{\tilde{p}, \gamma}\left([0, T] \rightarrow V_{2,0}\right)}^{\delta}\right] \\
& \stackrel{(3.26)-(3.28)}{\leq} C_{T}<\infty
\end{aligned}
$$


We conclude from (2) and (3) that

$$
\sup _{n} E^{W}\left[\left\|X^{n}\right\|_{\mathcal{I}}^{\delta}\right] \leq C_{T}<\infty
$$

and hence, that for $R>0$,

$$
\begin{aligned}
\sup _{n} P^{W}\left(\left\|X^{n}\right\|_{\mathcal{I}}>R\right) & \leq \frac{1}{R^{\delta}} \sup _{n} E^{W}\left[\left\|X_{.}^{n}\right\|_{\mathcal{I}}^{\delta}\right] \\
& \leq \frac{C_{T}}{R^{\delta}} \longrightarrow 0 \quad \text { as } R \longrightarrow \infty
\end{aligned}
$$

We will see from this and (2) that the set

$$
\left\{X . ;\left\|X^{n}\right\|_{\mathcal{I}} \leq R\right\}
$$

is relatively compact in $L_{\tilde{p}}\left([0, T] \rightarrow V_{\tilde{p}, 1}\right)$. Hence, by (4) we have the tightness (1).

Finally, Proposition 3.4.1 follows from Lemmas 3.4.3, 3.4.4 and the following:

\section{LEMMA 3.4.5. Suppose that:}

- $\left(S_{j}, \rho_{j}\right)(j=1, \ldots, m)$ are complete separable metric spaces such that all of $S_{j}(j=1, \ldots, m)$ are subsets of a set $S$;

- $\left(X_{n}\right)_{n \in \mathbb{N}}$ is a sequence of random variables with values in $\bigcap_{j=1}^{m} S_{j}$ defined on a probability space $(\Omega, \mathcal{F}, P)$;

- $\left(X_{n}\right)_{n \in \mathbb{N}}$ is tight in each of $\left(S_{j}, \rho_{j}\right), j=1, \ldots, m$, separately.

Then, there exists a sequence $n(k) \rightarrow \infty$, random variables $X, \widetilde{X}_{k}, k=$ $1,2, \ldots$, with values in $\bigcap_{j=1}^{m} S_{j}$ defined on a probability space $(\widetilde{\Omega}, \widetilde{\mathcal{F}}, \widetilde{P})$ such that

$$
\begin{aligned}
\widetilde{P}\left(\tilde{X}_{k} \in \cdot\right) & =P\left(X_{n(k)} \in\right) \quad \text { for all } k=1,2, \ldots ; \\
\lim _{k \rightarrow \infty} \sum_{j=1}^{m} \rho_{j}\left(X, \tilde{X}_{k}\right) & =0 \quad \tilde{P} \text {-a.s. }
\end{aligned}
$$

PROOF. By induction, it is enough to consider the case of $m=2$. Let $\varepsilon>0$ be arbitrary. Then, for $j=1,2$, there exists a compact subset $K_{j}$ of $S_{j}$ such that

$$
P\left(X_{n} \in K_{j}\right) \geq 1-\varepsilon \quad \text { for all } j=1,2 \text { and } n=1,2, \ldots
$$

Now a very simple but crucial observation is that $K_{1} \cap K_{2}$ is compact in $S_{1} \cap S_{2}$ with respect to the metric $\rho_{1}+\rho_{2}$. Also,

$$
P\left(X_{n} \in K_{1} \cap K_{2}\right) \geq 1-2 \varepsilon \quad \text { for all } j=1,2 \text { and } n=1,2, \ldots
$$

These imply that $\left(X_{n}\right)$ is tight in $S_{1} \cap S_{2}$ with respect to the metric $\rho_{1}+\rho_{2}$. Thus, the lemma follows from Prohorov's theorem ([1], Theorem 2.6, page 7) and Skorohod's representation theorem ([1], Theorem 2.7, page 9). 
REMARK. This remark, together with the one after Lemma 4.1.1, concerns the validity of Theorem 2.1 .3 with $p=2$ for all $d$. Let $\alpha<1$. Then we can also prove that

(3.29) the laws $\left\{P^{W}\left(X^{n} \in \cdot\right)\right\}_{n=1}^{\infty}$ are tight on $L_{p^{\prime \prime}, \text { loc }}\left([0, \infty) \rightarrow V_{p, \alpha}\right)$.

This can be seen as follows. We set

$$
\mathcal{I}=L_{p^{\prime \prime}}\left([0, T] \rightarrow V_{p, 1}\right) \cap L_{p^{\prime \prime}, \gamma}\left([0, T] \rightarrow V_{p^{\prime \prime},-\beta(p, 1)}\right), \quad \text { with } \gamma \in(0,1 / 2) .
$$

Since

$$
V_{p, 1} \stackrel{\text { compact }}{\hookrightarrow} V_{p, \alpha} \hookrightarrow V_{p^{\prime \prime},-\beta(p, 1)},
$$

we have by Lemma 3.3.3 that

$$
\mathcal{I}^{\text {compact }} L_{p^{\prime \prime}}\left([0, T] \rightarrow V_{p, \alpha}\right) .
$$

Then we get (3.29) by similar argument as in Lemma 3.4.4.

By the tightness (3.29), Lemmas 3.4.3 and 3.4.5, we obtain a variant of Proposition 3.4.1 in which the convergence $\widetilde{X}^{k} \rightarrow X, P$-a.s. takes place in the metric space

$$
C\left([0, \infty) \rightarrow V_{2 \wedge p^{\prime},-\beta}\right) \cap L_{p^{\prime \prime}, \operatorname{loc}}\left([0, \infty) \rightarrow V_{p, \alpha}\right)
$$

instead of (3.23). We note that this modification of Proposition 3.4.1 is valid for $p \in\left[\frac{2 d}{d+2}, \infty\right)$ since we did not use Lemma 3.2.2.

\section{Proof of Theorems 2.1.3 and 2.2.1.}

4.1. Proof of Theorem 2.1.3. Let $X$ and $\widetilde{X}^{k}$ be as in Proposition 3.4.1. We will verify (2.1) [with $\beta=\beta(p, 1)$ ] as well as (2.3)-(2.5) and (2.7) for $X$. (2.3) can easily be seen. In fact,

$$
\begin{aligned}
& \tilde{X}_{0}^{k} \rightarrow X_{0} \text { a.s. in } V_{2 \wedge p^{\prime},-\beta}, \\
& \widetilde{X}_{0}^{k} \stackrel{\text { law }}{=} X_{0}^{n(k)}=\mathcal{P}_{n(k)} \xi \rightarrow \xi \quad \text { in } V_{2,0} .
\end{aligned}
$$

Thus, the laws of $X_{0}$ and $\xi$ are identical.

$$
\tilde{X}_{0}^{k} \stackrel{\text { law }}{=} X_{0}^{n(k)}=\mathcal{P}_{n(k)} \xi \rightarrow \xi \quad \text { in } V_{2,0} .
$$

Note that the function

$$
v . \mapsto \sup _{t \leq T}\left\|v_{t}\right\|_{2}^{2}+\int_{0}^{T}\left\|v_{t}\right\|_{p, 1}^{p} d t
$$

is lower semi-continuous on the metric space (3.23). Thus, (2.7) follows from (3.10) and Proposition 3.4.1 via Fatou's lemma.

To show (2.4) and (2.5), we prepare the following: 
LEMMA 4.1.1. Let $\varphi \in \mathcal{V}$ and $T>0$. Then,

(4.1) $\lim _{k \rightarrow \infty} \int_{0}^{T}\left|\left\langle\varphi,\left(\tilde{X}_{t}^{k} \cdot \nabla\right) \tilde{X}_{t}^{k}-\left(X_{t} \cdot \nabla\right) X_{t}\right\rangle\right| d t=0 \quad$ in probability $(P)$,

$$
\begin{array}{ll}
\lim _{k \rightarrow \infty} \int_{0}^{T}\left|\left\langle e(\varphi), \tau\left(\tilde{X}_{t}^{k}\right)-\tau\left(X_{t}\right)\right\rangle\right| d t=0 & \text { in } L_{1}(P), \\
\lim _{k \rightarrow \infty} \int_{0}^{T}\left\langle\varphi, \mathcal{P}_{n(k)} b\left(\tilde{X}_{t}^{k}\right)-b\left(X_{t}\right)\right\rangle d t=0 & \text { in probability }(P) .
\end{array}
$$

PROOF. We write $Z_{t}^{k}=\tilde{X}_{t}^{k}-X_{t}$ to simplify the notation. We start by proving that

$$
\lim _{k \rightarrow \infty} E\left[\int_{0}^{T}\left\|Z_{t}^{k}\right\|_{p_{1}, 1}^{p_{1}} d t\right]=0, \quad \text { if } p_{1}<p .
$$

By Proposition 3.4.1,

$$
I_{k} \stackrel{\text { def. }}{=} \int_{0}^{T}\left\|Z_{t}^{k}\right\|_{1,1} d t \stackrel{k \rightarrow \infty}{\longrightarrow} 0, \quad P \text {-a.s. }
$$

Moreover, the random variables $\left\{I_{k}\right\}_{k \geq 1}$ are uniformly integrable since

$$
E\left[I_{k}^{p}\right] \stackrel{(3.10)}{\leq} C_{T}<\infty .
$$

Therefore,

$$
\lim _{k \rightarrow \infty} E\left[I_{k}\right]=0
$$

Let $k(m) \nearrow \infty$ be such that

$$
\Phi_{m, t} \stackrel{\text { def. }}{=}\left|Z_{t}^{k(m)}\right|+\left|\nabla Z_{t}^{k(m)}\right| \stackrel{m \rightarrow \infty}{\longrightarrow} 0,\left.\quad d t\right|_{[0, T]} \times d x \times P \text {-a.e., }
$$

where $\left.d t\right|_{[0, T]} \times d x$ denotes the Lebesgue measure on $[0, T] \times \mathbb{T}^{d}$. Such a sequence $k(m)$ exists by (2). The sequence $\left\{\Phi_{m},\right\}_{m \geq 1}$ is uniformly integrable with respect to $\left.d t\right|_{[0, T]} \times d x \times P$. In fact,

$$
E\left[\int_{0}^{T} \int_{\mathbb{T}^{d}} \Phi_{m, t}^{p} d t\right] \stackrel{(3.10)}{\leq} C_{T}<\infty .
$$

Therefore, (3), together with this uniform integrability, implies (4.4) along the subsequence $k(m)$. Finally, we get rid of the subsequence, since the subsequence as $k(m)$ above can be chosen from any subsequence of $k$ given in advance. We now prove (4.1). Since

$$
\left(\tilde{X}_{t}^{k} \cdot \nabla\right) \tilde{X}_{t}^{k}-\left(X_{t} \cdot \nabla\right) X_{t}=\left(Z_{t}^{k} \cdot \nabla\right) \tilde{X}_{t}^{k}+\left(X_{t} \cdot \nabla\right) Z_{t}^{k},
$$

we have

$$
\int_{0}^{T}\left|\left\langle\varphi,\left(\tilde{X}_{t}^{k} \cdot \nabla\right) \tilde{X}_{t}^{k}-\left(X_{t} \cdot \nabla\right) X_{t}\right\rangle\right| d t \leq J_{1}+J_{2}
$$


where

$$
J_{1}=\int_{0}^{T}\left|\left\langle\varphi,\left(Z_{t}^{k} \cdot \nabla\right) \tilde{X}_{t}^{k}\right\rangle\right| d t \quad \text { and } \quad J_{2}=\int_{0}^{T}\left|\left\langle\varphi,\left(X_{t} \cdot \nabla\right) Z_{t}^{k}\right\rangle\right| d t .
$$

We may take $p_{1}$ in (4.4) as bigger than $\frac{3 d}{d+2}$ so that there exists $0<\alpha<1$ such that $\frac{2 d}{d+2 \alpha}<p_{1}$. Then by (1.25), we have that

$$
\left|\left\langle\varphi,\left(Z_{t}^{k} \cdot \nabla\right) \tilde{X}_{t}^{k}\right\rangle\right| \leq C\left\|Z_{t}^{k}\right\|_{p_{1}, \alpha}\left\|\tilde{X}_{t}^{k}\right\|_{2}\|\varphi\|_{p_{1}, \beta\left(p_{1}, \alpha\right)}
$$

and hence that

$$
J_{1} \leq C\|\varphi\|_{p_{1}, \beta\left(p_{1}, \alpha\right)} \sup _{t \leq T}\left\|\widetilde{X}_{t}^{k}\right\|_{2} \int_{0}^{T}\left\|Z_{t}^{k}\right\|_{p_{1}, \alpha} d t .
$$

By (3.10) and (4.4),

$$
\sup _{k \geq 1} E\left[\sup _{t \leq T}\left\|\tilde{X}_{t}^{k}\right\|_{2}^{2}\right]<\infty \quad \text { and } \quad \lim _{k \rightarrow \infty} \int_{0}^{T}\left\|Z_{t}^{k}\right\|_{p_{1}, \alpha} d t=0 \quad P \text {-a.s. }
$$

Thus, $\lim _{k \rightarrow \infty} J_{1}=0$ in probability. On the other hand, we have by (1.28) that

$$
\left|\left\langle\varphi,\left(X_{t} \cdot \nabla\right) Z_{t}^{k}\right\rangle\right| \leq C\left\|Z_{t}^{k}\right\|_{p_{1}, \alpha}\left\|X_{t}\right\|_{2}\|\varphi\|_{p_{1}, \beta\left(p_{1}, \alpha\right)}
$$

and hence that

$$
J_{2} \leq C\|\varphi\|_{p_{1}, \beta\left(p_{1}, \alpha\right)} \sup _{t \leq T}\left\|X_{t}\right\|_{2} \int_{0}^{T}\left\|Z_{t}^{k}\right\|_{p_{1}, \alpha} d t .
$$

By (2.7) and (4.4),

$$
E\left[\sup _{t \leq T}\left\|X_{t}\right\|_{2}^{2}\right]<\infty \quad \text { and } \quad \lim _{k \rightarrow \infty} \int_{0}^{T}\left\|Z_{t}^{k}\right\|_{p_{1}, \alpha} d t=0 \quad P \text {-a.s. }
$$

Thus, $\lim _{k \rightarrow \infty} J_{2}=0$ in probability.

We now turn to (4.2). It is enough to prove that

$$
\lim _{k \rightarrow \infty} E\left[\int_{0}^{T}\left\|\tau\left(\tilde{X}_{t}^{k}\right)-\tau\left(X_{t}\right)\right\|_{1} d t\right]=0
$$

Again, let $k(m)$ be such that (3) holds. Then,

$$
\lim _{m \rightarrow \infty} \tau\left(\widetilde{X}_{t}^{k(m)}\right)=\tau\left(X_{t}\right),\left.\quad d t\right|_{[0, T]} \times d x \times P \text {-a.e. }
$$

On the other hand, we have for $p^{\prime}=\frac{p}{p-1}$ that

$$
E\left[\int_{0}^{T} d t \int_{\mathbb{T}^{d}}\left|\tau\left(\tilde{X}_{t}^{k}\right)\right|^{p^{\prime}}\right] \leq C E\left[\int_{0}^{T} d t \int_{\mathbb{T}^{d}}\left(1+\left|e\left(\tilde{X}_{t}^{k}\right)\right|\right)^{p}\right] \stackrel{(3.10)}{\leq} C_{T}<\infty,
$$

which implies that $\tau\left(\tilde{X}_{t}^{k}\right), k \in \mathbb{N}$ are uniformly integrable with respect to $\left.d t\right|_{[0, T]} \times$ $d x \times P$. Therefore, (5), together with this uniform integrability, implies (4) along 
the subsequence $k(m)$. Finally, we get rid of the subsequence, since the subsequence as $k(m)$ above can be chosen from any subsequence of $k$ given in advance.

Equation (4.3) follows from (4.1) and (4.2). Since $\varphi \in \mathcal{V}$ is fixed and $k$ is tending to $\infty$, we do not have to care about $\mathcal{P}_{n(k)}$ here.

REMARK. If $p=2$, then Lemma 4.1.1 is valid for all $d$. This is for the following reason. By inspection of the proof above, we see immediately that (4.1) follows also from the modification of Proposition 3.4.1 mentioned at the end of Section 3.4. Also, for $p=2,(4.2)$ is equivalent to

$$
\lim _{k \rightarrow \infty} \int_{0}^{T}\left\langle\Delta \varphi, \widetilde{X}_{t}^{k}-X_{t}\right\rangle d t=0 \quad \text { in } L_{1}(P),
$$

which also follows from the modification of Proposition 3.4.1 mentioned at the end of Section 3.4.

\section{LEMMA 4.1.2. Let}

$$
Y_{t}=Y_{t}(X)=X_{t}-X_{0}-\int_{0}^{t} b\left(X_{s}\right) d s, \quad t \geq 0 .
$$

Then, $Y$. is a $\operatorname{BM}\left(V_{2,0}, \Gamma\right)$. Moreover, $Y_{t+} .-Y_{t}$ and $\left\{\left\langle\varphi, X_{s}\right\rangle ; s \leq t, \varphi \in \mathcal{V}\right\}$ are independent for any $t \geq 0$.

PROOF. It is enough to prove that for each $\varphi \in \mathcal{V}$ and $0 \leq s<t$,

$$
E\left[\exp \left(\mathbf{i}\left\langle\varphi, Y_{t}-Y_{s}\right\rangle\right) \mid \mathcal{G}_{s}\right]=\exp \left(-\frac{t-s}{2}\langle\varphi, \Gamma \varphi\rangle\right), \quad \text { a.s. }
$$

where $\mathcal{G}_{s}=\sigma\left(\left\langle\varphi, X_{u}\right\rangle ; u \leq s, \varphi \in \mathcal{V}\right)$. We set

$$
F(X)=f\left(\left\langle\varphi_{1}, X_{u_{1}}\right\rangle, \ldots,\left\langle\varphi_{n}, X_{u_{n}}\right\rangle\right),
$$

where $f \in C_{\mathrm{b}}\left(\mathbb{R}^{n}\right), 0 \leq u_{1}<\cdots<u_{n} \leq s$ and $\varphi_{1}, \ldots, \varphi_{n} \in \mathcal{V}$ are chosen arbitrarily in advance. Then (1) can be verified by showing that

$$
E\left[\exp \left(\mathbf{i}\left\langle\varphi, Y_{t}-Y_{s}\right\rangle\right) F(X)\right]=\exp \left(-\frac{t-s}{2}\langle\varphi, \Gamma \varphi\rangle\right) E[F(X)] .
$$

Let

$$
Y_{t}^{k}=\tilde{X}_{t}^{k}-\tilde{X}_{0}^{k}-\int_{0}^{t} \mathcal{P}_{n(k)} b\left(\tilde{X}_{s}^{k}\right) d s, \quad t \geq 0 .
$$

Then we see from Theorem 3.1.1 that

$$
\begin{aligned}
& E\left[\exp \left(\mathbf{i}\left\langle\varphi, Y_{t}^{k}-Y_{s}^{k}\right\rangle\right) F\left(\tilde{X}^{k}\right)\right] \\
& \quad=\exp \left(-\frac{t-s}{2}\left\langle\varphi, \Gamma \mathcal{P}_{n(k)} \varphi\right\rangle\right) E\left[F\left(\tilde{X}^{k}\right)\right] .
\end{aligned}
$$


Moreover, we have

$$
\lim _{k \rightarrow \infty}\left\langle\varphi, Y_{t}^{k}-Y_{s}^{k}\right\rangle \stackrel{(3.24),(4.3)}{=} \lim _{k \rightarrow \infty}\left\langle\varphi, Y_{t}-Y_{s}\right\rangle \quad \text { in probability }
$$

and hence,

$$
\lim _{k \rightarrow \infty} \operatorname{LHS} \text { of }(3)=\text { LHS of (2). }
$$

On the other hand,

$$
\lim _{k \rightarrow \infty} \operatorname{RHS} \text { of (3) } \stackrel{(3.24)}{=} \text { RHS of (2). }
$$

These prove (2).

Finally, we prove (2.1) with $\beta=\beta(p, 1)$. It follows from (2.7) that

$$
X \in L_{p, \operatorname{loc}}\left([0, \infty) \rightarrow V_{p, 1}\right) \cap L_{\infty, \operatorname{loc}}\left([0, \infty) \rightarrow V_{2,0}\right)
$$

Thus, it remains to show that $X \in C\left([0, \infty) \rightarrow V_{2 \wedge p^{\prime},-\beta(p, 1)}\right)$. But this follows from Lemma 3.2.1 and that $Y \in C\left([0, \infty) \rightarrow V_{2,0}\right)$.

4.2. Proof of Theorem 2.2.1. Here we can follow the argument of [5], Theorem 4.29, page 254, almost verbatim. We will present it for the convenience of the readers.

We need two technical lemmas.

LEMMA 4.2.1. Let $H$ be a Hilbert space and $V$ be a Banach space such that

$$
V \hookrightarrow H \hookrightarrow V^{*}
$$

Suppose that $f \in L_{p}([0, T] \rightarrow V)(p \in(1, \infty), T>0)$ has derivative $f^{\prime}$ in $L_{p^{\prime}}\left([0, T] \rightarrow V^{*}\right)$. Then,

$$
\frac{d}{d t}|f|_{H}^{2}=2_{V}\left\langle f, f^{\prime}\right\rangle_{V^{*}}
$$

in the distributional sense on $(0, T)$.

PROOF. The case of $p=2$ can be found in [7], Lemma 1.2, pages 60-61. The extension to general $p$ is straightforward.

LEMMA 4.2.2 ([5], Lemma 4.35, page 255). Let $q \in(2, \infty)$ if $d=2$ and $q \in\left[2, \frac{2 d}{d-2}\right]$ if $d \geq 3$. Then there exists $c \in(0, \infty)$ such that

$$
\|v\|_{q} \leq c\|v\|_{2}^{\theta}\|\nabla v\|_{2}^{1-\theta} \quad \text { with } \theta=\frac{2 d-q(d-2)}{2 q}
$$

for all $v \in V_{2,1}$ with $\int_{\mathbb{T}^{d}} v=0$. 
Let $X$ and $\tilde{X}$ be as in the assumptions of Theorem 2.2.1 and

$$
Z_{t}=X_{t}-\tilde{X}_{t}=\int_{0}^{t}\left(b\left(X_{s}\right)-b\left(\tilde{X}_{s}\right)\right) d s
$$

Then,

$$
Z . \in L_{p, \operatorname{loc}}\left([0, \infty) \rightarrow V_{p, 1}\right)
$$

and by Lemma 3.2.1,

$$
\partial_{t} Z .=b(X .)-b(\tilde{X} .) \in L_{p, \operatorname{loc}}\left([0, \infty) \rightarrow V_{p^{\prime},-\beta(p, 1)}\right) .
$$

Since $p \geq p^{\prime}$ and $\beta(p, 1)=1$ for $p \geq 1+\frac{d}{2}\left(\geq \frac{4 d}{d+2}\right)$, we see from (2) and Lemma 4.2.1 (applied to $f=Z$. and $V=V_{p, 1}$ ) that

$$
\frac{1}{2} \frac{d}{d t}\left\|Z_{t}\right\|_{2}^{2} \stackrel{(4.6)}{=}\left\langle Z_{t}, b\left(X_{t}\right)-b\left(\tilde{X}_{t}\right)\right\rangle=-I_{t}-J_{t}
$$

in the distributional sense, where

$$
I_{t}=\left\langle Z_{t},\left(X_{t} \cdot \nabla\right) X_{t}-\left(\tilde{X}_{t} \cdot \nabla\right) \tilde{X}_{t}\right\rangle \quad \text { and } \quad J_{t}=\left\langle e\left(Z_{t}\right), \tau\left(X_{t}\right)-\tau\left(\tilde{X}_{t}\right)\right\rangle .
$$

We have by [5], formula (1.25), page 198 and formula (1.11), page 196, that

$$
J_{t} \geq c_{1}\left\|e\left(Z_{t}\right)\right\|_{2}^{2} \geq c_{2}\left\|\nabla Z_{t}\right\|_{2}^{2} .
$$

On the other hand, since $\tilde{X}_{t}=X_{t}-Z_{t}$, we see that

$$
\left\langle Z_{t},\left(\tilde{X}_{t} \cdot \nabla\right) \tilde{X}_{t}\right\rangle \stackrel{(1.18)}{=}\left\langle Z_{t},\left(\tilde{X}_{t} \cdot \nabla\right) X_{t}\right\rangle=\left\langle Z_{t},\left(\left(X_{t}-Z_{t}\right) \cdot \nabla\right) X_{t}\right\rangle,
$$

and hence that

$$
I_{t}=\left\langle Z_{t},\left(Z_{t} \cdot \nabla\right) X_{t}\right\rangle
$$

Therefore,

$$
\begin{aligned}
& \left|I_{t}\right|^{1 / p+(p-1) /(2 p)+(p-1) /(2 p)=1}\left\|\nabla X_{t}\right\|_{p}\left\|Z_{t}\right\|_{2 p /(p-1)}^{2} \\
& \stackrel{(4.7)}{\leq} C_{3}\left\|\nabla Z_{t}\right\|_{2}^{d / p}\left\|\nabla X_{t}\right\|_{p}\left\|Z_{t}\right\|_{2}^{(2 p-d) / p} \\
& d /(2 p)+(2 p-d) /(2 p)=1 \quad c_{2}\left\|\nabla Z_{t}\right\|_{2}^{2}+C_{4}\left\|\nabla X_{t}\right\|_{p}^{2 p /(2 p-d)}\left\|Z_{t}\right\|_{2}^{2} .
\end{aligned}
$$

We see from (3)-(5) that

$$
\frac{1}{2} \frac{d}{d t}\left\|Z_{t}\right\|_{2}^{2} \leq C_{4}\left\|\nabla X_{t}\right\|_{p}^{2 p /(2 p-d)}\left\|Z_{t}\right\|_{2}^{2} .
$$

Since $\frac{2 p}{2 p-d} \leq p$, this implies via Gronwall's lemma (we need an appropriate generalization since the derivative above is in the distributional sense) that

$$
\left\|Z_{t}\right\|_{2}^{2} \leq\left\|Z_{0}\right\|_{2}^{2} \exp \left(C_{4} \int_{0}^{t}\left\|\nabla X_{s}\right\|_{p}^{2 p /(2 p-d)} d s\right) .
$$

This proves that $\left\|Z_{t}\right\|_{2} \equiv 0$. 
Acknowledgments. The authors thank Professor Josef Málek for valuable comments on the earlier version of this article. N. Yoshida thanks Professor Kenji Nakanishi for useful conversations.

\section{REFERENCES}

[1] Ikeda, N. and Watanabe, S. (1989). Stochastic Differential Equations and Diffusion Processes, 2nd ed. North-Holland Mathematical Library 24. North-Holland, Amsterdam. MR1011252

[2] Flandoli, F. (2008). An introduction to 3D stochastic fluid dynamics. In SPDE in Hydrodynamic: Recent Progress and Prospects. Lecture Notes in Math. 1942 51-150. Springer, Berlin. MR2459085

[3] Flandoli, F. and GatareK, D. (1995). Martingale and stationary solutions for stochastic Navier-Stokes equations. Probab. Theory Related Fields 102 367-391. MR1339739

[4] Grafakos, L. (2004). Classical and Modern Fourier Analysis. Pearson Education, Upper Saddle River, NJ. MR2449250

[5] MÁlek, J., NeČAs, J., RokytA, M. and RÅUŽIČKA, M. (1996). Weak and Measure-Valued Solutions to Evolutionary PDEs. Applied Mathematics and Mathematical Computation 13. Chapman and Hall, London. MR1409366

[6] TaYlor, M. E. (1996). Partial Differential Equations. III. Applied Mathematical Sciences 116. Springer, New York. MR1395149

[7] Temam, R. (1979). Navier-Stokes Equations, Revised ed. Studies in Mathematics and Its Applications 2. North-Holland, Amsterdam. MR0603444

[8] YoshidA, N. (2010). Stochastic shear thickening fluids: Strong convergence of the Galerkin approximation and the energy equality. Preprint. Available at: arXiv:1009.2136.

Graduate SCHOOL OF Mathematical SCIENCES

UNIVERSITY OF TOKYO

Komaba, Meguro-Ku TOKYO 153-8914

JAPAN

E-MAIL: yutaka@ms.u-tokyo.ac.jp
DIVISION OF MATHEMATICS

GRADUATE SCHOOL OF SCIENCE

KYOTO UNIVERSITY

KYOTO 606-8502

JAPAN

E-MAIL: nobuo@math.kyoto-u.ac.jp

URL: http://www.math.kyoto-u.ac.jp/ nobuo/ 